\title{
ON THE DISCRETE LOGARITHMIC MINKOWSKI PROBLEM
}

\author{
KÁROLY J. BÖRÖCZKY, PÁL HEGEDÜS, AND GUANGXIAN ZHU
}

\begin{abstract}
A new sufficient condition for the existence of a solution for the logarithmic Minkowski problem is established. This new condition contains the one established by Zhu [69] and the discrete

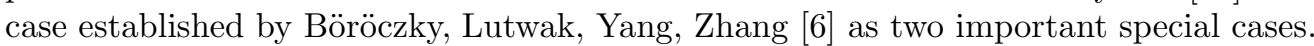

\section{INTRODUCTION}

The setting for this paper is $n$-dimensional Euclidean space $\mathbb{R}^{n}$. A convex body in $\mathbb{R}^{n}$ is a compact convex set that has non-empty interior. If $K$ is a convex body in $\mathbb{R}^{n}$, then the surface area measure, $S_{K}$, of $K$ is a Borel measure on the unit sphere, $S^{n-1}$, defined for a Borel $\omega \subset S^{n-1}$ (see, e.g., Schneider [61]), by

$$
S_{K}(\omega)=\int_{x \in \nu_{K}^{-1}(\omega)} d \mathcal{H}^{n-1}(x),
$$

where $\nu_{K}: \partial^{\prime} K \rightarrow S^{n-1}$ is the Gauss map of $K$, defined on $\partial^{\prime} K$, the set of points of $\partial K$ that have a unique outer unit normal, and $\mathcal{H}^{n-1}$ is $(n-1)$-dimensional Hausdorff measure.

As one of the cornerstones of the classical Brunn-Minkowski theory, the Minkowski's existence theorem can be stated as follows (see, e.g., Schneider [61]): If $\mu$ is not concentrated on a great subsphere of $S^{n-1}$, then $\mu$ is the surface area measure of a convex body if and only if

$$
\int_{S^{n-1}} u d \mu(u)=0
$$

The solution is unique up to translation, and even the regularity of the solution is well investigated, see e.g., Lewy [40], Nirenberg [57], Cheng and Yau [12], Pogorelov [60], and Caffarelli [9].

The surface area measure of a convex body has clear geometric significance. Another important measure that is associated with a convex body and that has clear geometric importance is the cone-volume measure. If $K$ is a convex body in $\mathbb{R}^{n}$ that contains the origin in its interior, then the cone-volume measure, $V_{K}$, of $K$ is a Borel measure on $S^{n-1}$ defined for each Borel $\omega \subset S^{n-1}$ by

$$
V_{K}(\omega)=\frac{1}{n} \int_{x \in \nu_{K}^{-1}(\omega)} x \cdot \nu_{K}(x) d \mathcal{H}^{n-1}(x) .
$$

For references regarding cone-volume measure see, e.g., [5, 8, 42, 44, 55, 56, 58, 62, 64, 69,

The Minkowski's existence theorem deals with the question of prescribing the surface area measure. The following problem is prescribing the cone-volume measure.

Logarithmic Minkowski problem: What are the necessary and sufficient conditions on a finite Borel measure $\mu$ on $S^{n-1}$ so that $\mu$ is the cone-volume measure of a convex body in $\mathbb{R}^{n}$ ?

In [45], Lutwak showed that there is an $L_{p}$ analogue of the surface area measure (known as the $L_{p}$ surface area measure). In recent years, the $L_{p}$ surface area measure appeared in, e.g.,

2010 Mathematics Subject Classification: 52A40.

Key Words: Polytope, cone-volume measure, Minkowski problem, $L_{p}$ Minkowski problem, logarithmic Minkowski problem, Monge-Ampère equation. 
$[1,4,10,22,23,25,26,31,42,44,47,-49,52,53,55,56,58,59,64$. In [45, Lutwak posed the associated $L_{p}$ Minkowski problem which extends the classical Minkowski problem for $p \geq 1$. In addition, the $L_{p}$ Minkowski problem for $p<1$ was publicized by a series of talks by Erwin Lutwak in the 1990's. The $L_{p}$ Minkowski problem is the classical Minkowski problem when $p=1$, while the $L_{p}$ Minkowski problem is the logarithmic Minkowski problem when $p=0$. The $L_{p}$ Minkowski problem is interesting for all real $p$, and have been studied by, e.g., Lutwak [45], Lutwak and Oliker [46], Chou and Wang [14, Guan and Lin [21], Hug, et al. [35], Böröczky, et al. [6]. Additional references regarding the $L_{p}$ Minkowski problem and Minkowski-type problems can be found in, e.g., [6, 11, 14, 20 24, 33 35, 38, 39, 41, 45, 46, 51, 54, 62, 63, 70, 71]. Applications of the solutions to the $L_{p}$ Minkowski problem can be found in, e.g., [2, 3, 13, 15, 16, 27, 29, 36, 37, 50, 66, 68, .

A finite Borel measure $\mu$ on $S^{n-1}$ is said to satisfy the subspace concentration condition if, for every subspace $\xi$ of $\mathbb{R}^{n}$, such that $0<\operatorname{dim} \xi<n$,

$$
\mu\left(\xi \cap S^{n-1}\right) \leq \frac{\operatorname{dim} \xi}{n} \mu\left(S^{n-1}\right),
$$

and if equality holds in (1.2) for some subspace $\xi$, then there exists a subspace $\xi^{\prime}$, that is complementary to $\xi$ in $\mathbb{R}^{n}$, so that also

$$
\mu\left(\xi^{\prime} \cap S^{n-1}\right)=\frac{\operatorname{dim} \xi^{\prime}}{n} \mu\left(S^{n-1}\right) .
$$

The measure $\mu$ on $S^{n-1}$ is said to satisfy the strict subspace concentration inequality if the inequality in (1.2) is strict for each subspace $\xi \subset \mathbb{R}^{n}$, such that $0<\operatorname{dim} \xi<n$.

Very recently, Böröczky and Henk [5] proved that if the centroid of a convex body is the origin, then the cone-volume measure of this convex body satisfies the subspace concentration condition. For more references on the progress of the subspace concentration condition, see, e.g., Henk et al. [32, He et al. [30, Xiong [67], Böröczky et al. [8], and Henk and Linke [31].

In [6], Böröczky, et al. established the following necessary and sufficient conditions for the existence of solutions to the even logarithmic Minkowski problem.

Theorem 1.1 (Böröczky,Lutwak,Yang,Zhang). A non-zero finite even Borel measure on $S^{n-1}$ is the cone-volume measure of an origin-symmetric convex body in $\mathbb{R}^{n}$ if and only if it satisfies the subspace concentration condition.

The convex hull of a finite set is called a polytope provided that it has positive $n$-dimensional volume. The convex hull of a subset of these points is called a facet of the polytope if it lies entirely on the boundary of the polytope and has positive $(n-1)$-dimensional volume. If a polytope $P$ contains the origin in its interior and has $N$ facets whose outer unit normals are $u_{1}, \ldots, u_{N}$, and such that if the facet with outer unit normal $u_{k}$ has $(n-1)$-measure $a_{k}$ and distance from the origin $h_{k}$ for all $k \in\{1, \ldots, N\}$, then

$$
V_{P}=\frac{1}{n} \sum_{k=1}^{N} h_{k} a_{k} \delta_{u_{k}} .
$$

where $\delta_{u_{k}}$ denotes the delta measure that is concentrated at the point $u_{k}$.

A finite subset $U$ (with no less than $n$ elements) of $S^{n-1}$ is said to be in general position if any $k$ elements of $U, 1 \leq k \leq n$, are linearly independent.

For a long time, people believed that the data for a cone-volume measure can not be arbitrary. However, Zhu [69] proved that any discrete measure on $S^{n-1}$ whose support is in general position is a cone-volume measure. 
Theorem $1.2(\mathrm{Zhu})$. A discrete measure, $\mu$, on the unit sphere $S^{n-1}$ is the cone-volume measure of a polytope whose outer unit normals are in general position if and only if the support of $\mu$ is in general position and not concentrated on a closed hemisphere of $S^{n-1}$.

A linear subspace $\xi(1 \leq \operatorname{dim} \xi \leq n-1)$ of $\mathbb{R}^{n}$ is said to be essential with respect to a Borel measure $\mu$ on $S^{n-1}$ if $\xi \cap \operatorname{supp}(\mu)$ is not concentrated on any closed hemisphere of $\xi \cap S^{n-1}$.

Definition 1.3. A finite Borel measure $\mu$ on $S^{n-1}$ is said to satisfy the essential subspace concentration condition if, for every essential subspace $\xi$ (with respect to $\mu$ ) of $\mathbb{R}^{n}$, such that $0<\operatorname{dim} \xi<$ $n$,

$$
\mu\left(\xi \cap S^{n-1}\right) \leq \frac{\operatorname{dim} \xi}{n} \mu\left(S^{n-1}\right),
$$

and if equality holds in (1.3) for some essential subspace $\xi$ (with respect to $\mu$ ), then there exists a subspace $\xi^{\prime}$, that is complementary to $\xi$ in $\mathbb{R}^{n}$, so that

$$
\mu\left(\xi^{\prime} \cap S^{n-1}\right)=\frac{\operatorname{dim} \xi^{\prime}}{n} \mu\left(S^{n-1}\right) .
$$

Definition 1.4. The measure $\mu$ on $S^{n-1}$ is said to satisfy the strict essential subspace concentration inequality if the inequality in (1.3) is strict for each essential subspace $\xi$ (with respect to $\mu$ ) of $\mathbb{R}^{n}$, such that $0<\operatorname{dim} \xi<n$.

We would like to note that if $\mu$ is a Borel measure on the unit sphere that is not concentrated on a closed hemisphere and satisfies the essential subspace concentration condition, and $\xi$ is an essential subspace (with respect to $\mu$ ) that reaches the equality in (1.3), then by Lemma 5.2, $\xi^{\prime}$ (in (1.4)) is an essential subspace with respect to $\mu$.

It is the aim of this paper to establish the following.

Theorem 1.5. If $\mu$ is a discrete measure on $S^{n-1}$ that is not concentrated on any closed hemisphere and satisfies the essential subspace concentration condition, then $\mu$ is the cone-volume measure of a polytope in $\mathbb{R}^{n}$ containing the origin in its interior.

If $\mu$ is a non-trivial even Borel measure on $S^{n-1}$, and $\xi$ is a $k$-dimensional linear subspace of $\mathbb{R}^{n}$ spanned by some vectors $v_{1}, \ldots, v_{k} \in \operatorname{supp}(\mu)$ for $1 \leq k \leq n-1$, then $-v_{1}, \ldots,-v_{k} \in$ $\operatorname{supp}(\mu)$, as well, and hence $\xi$ is an essential subspace. In particular, for even discrete measures, Theorem 1.5 is equivalent to the sufficient condition of Theorem 1.1. However, there are non-even discrete measures that satisfy the essential subspace concentration condition, but not the subspace concentration condition. For example, if a $k$-dimensional subspace $\xi, 1 \leq k \leq n-1$, intersects the support of the measure in $k+1$ unit vectors $u_{0}, \ldots, u_{k}$ such that $u_{1}, \ldots, u_{k}$ are independent, and $u_{0}=\alpha_{1} u_{1}+\ldots+\alpha_{k} u_{k}$ for $\alpha_{1}, \ldots, \alpha_{k}>0$, then there is no condition on the restriction of the measure to $\xi \cap S^{n-1}$. Therefore, for discrete measures, Theorem 1.5 is a generalization of the sufficient condition of Theorem 1.1.

We claim that if the support of a discrete measure $\mu$ is in general position, then the set of essential subspaces (with respect to $\mu$ ) is empty. Otherwise, there exists a subspace $\xi$ with $1 \leq \operatorname{dim} \xi \leq n-1$ such that $\operatorname{supp}(\mu) \cap \xi$ is not concentrated on a closed hemisphere of $S^{n-1} \cap \xi$. Then we can choose $\operatorname{dim} \xi+1(\leq n)$ vectors from $\operatorname{supp}(\mu) \cap \xi$ that are linearly dependent. But this contradicts the fact that $\operatorname{supp}(\mu)$ is in general position. From our declaration, we have, Theorem 1.5 contains Theorem 1.2 as an important special case.

In $\mathbb{R}^{2}$, Theorem [1.5 leads to the main result of Stancu ( [62], pp. 162), where she applied a different method called the crystalline deformation.

New inequalities for cone-volume measures are established in section [6, 


\section{Preliminaries}

In this section, we collect some basic notations and facts about convex bodies. For general references regarding convex bodies see, e.g., [17-19,61, 65].

The vectors of this paper are column vectors. For $x, y \in \mathbb{R}^{n}$, we will write $x \cdot y$ for the standard inner product of $x$ and $y$, and write $|x|$ for the Euclidean norm of $x$. We write $S^{n-1}=\left\{x \in \mathbb{R}^{n}\right.$ : $|x|=1\}$ for the boundary of the Euclidean unit ball $B^{n}$ in $\mathbb{R}^{n}$, and write $\kappa_{n}$ for the volume of the unit ball. Let $V_{k}(M)$ denote the $k$-dimensional Hausdorff measure of an at most $k$-dimensional convex set $M$. In addition, if $k=n-1$, then we also use the notation $|M|$.

Suppose $X_{1}, X_{2}$ are subspaces of $\mathbb{R}^{n}$, we write $X_{1} \perp X_{2}$ if $x_{1} \cdot x_{2}=0$ for all $x \in X_{1}$ and $x_{2} \in X_{2}$. Suppose $X$ is a subspace of $\mathbb{R}^{n}$ and $S$ is a subset of $\mathbb{R}^{n}$, we write $\left.S\right|_{X}$ for the orthogonal projection of $S$ on $X$.

Suppose $C$ is a subset of $\mathbb{R}^{n}$, the positive hull, $\operatorname{pos}(C)$, of $C$ is the set of all positive combinations of any finitely many elements of $C$. Let $\operatorname{lin}(C)$ be the smallest linear subspace of $\mathbb{R}^{n}$ containing $C$. The diameter of $C$ is defined by

$$
d(C)=\sup \{|x-y|: x, y \in C\} .
$$

For $K_{1}, K_{2} \subset \mathbb{R}^{n}$ and $c_{1}, c_{2} \geq 0$, the Minkowski combination, $c_{1} K_{1}+c_{2} K_{2}$, is defined by

$$
c_{1} K_{1}+c_{2} K_{2}=\left\{c_{1} x_{1}+c_{2} x_{2}: x_{1} \in K_{1}, x_{2} \in K_{2}\right\} .
$$

The support function $h_{K}: \mathbb{R}^{n} \rightarrow \mathbb{R}$ of a compact convex set $K$ is defined, for $x \in \mathbb{R}^{n}$, by

$$
h(K, x)=\max \{x \cdot y: y \in K\} .
$$

Obviously, for $c \geq 0$ and $x \in \mathbb{R}^{n}$, we have

$$
h(c K, x)=h(K, c x)=\operatorname{ch}(K, x) .
$$

The convex hull of two convex sets $K, L$ in $\mathbb{R}^{n}$ is defined by

$$
[K, L]=\{z: z=\lambda x+(1-\lambda) y, 0 \leq \lambda \leq 1 \text { and } x, y \in K \cup L\} .
$$

The Hausdorff distance of two compact sets $K, L$ in $\mathbb{R}^{n}$ is defined by

$$
\delta(K, L)=\inf \left\{t \geq 0: K \subset L+t B^{n}, L \subset K+t B^{n}\right\} .
$$

It is known that the Hausdorff distance between two convex bodies, $K$ and $L$, is

$$
\delta(K, L)=\max _{u \in S^{n-1}}|h(K, u)-h(L, u)| .
$$

We always consider the space of convex bodies as metric space equipped with the Hausdorff distance. It is known that if a sequence $\left\{K_{m}\right\}$ of convex bodies tends to a convex body $K$ in $\mathbb{R}^{n}$ containing the origin in its interior, then $S_{K_{m}}$ tends weakly to $S_{K}$, and hence $V_{K_{m}}$ tends weakly to $V_{K}$ (see Schneider [61]).

For a convex body $K$ in $\mathbb{R}^{n}$, and $u \in S^{n-1}$, the support hyperplane $H(K, u)$ in direction $u$ is defined by

$$
H(K, u)=\left\{x \in \mathbb{R}^{n}: x \cdot u=h(K, u)\right\},
$$

the face $F(K, u)$ in direction $u$ is defined by

$$
F(K, u)=K \cap H(K, u) .
$$

Let $\mathcal{P}$ be the set of all polytopes in $\mathbb{R}^{n}$. If the unit vectors $u_{1}, \ldots, u_{N}$ are not concentrated on a closed hemisphere, let $\mathcal{P}\left(u_{1}, \ldots, u_{N}\right)$ be the set of all polytopes $P \in \mathcal{P}$ such that the set of outer unit normals of the facets of $P$ is a subset of $\left\{u_{1}, \ldots, u_{N}\right\}$, and let $\mathcal{P}_{N}\left(u_{1}, \ldots, u_{N}\right)$ be the the set of all polytopes $P \in \mathcal{P}$ such that the set of outer unit normals of the facets of $P$ is $\left\{u_{1}, \ldots, u_{N}\right\}$. 


\section{An extremal problem Related to the logarithmic Minkowski Problem}

Let us suppose $\gamma_{1}, \ldots, \gamma_{N} \in(0, \infty)$, and the unit vectors $u_{1}, \ldots, u_{N}$ are not concentrated on a closed hemisphere. Let

$$
\mu=\sum_{i=1}^{N} \gamma_{i} \delta_{u_{i}}
$$

and for $P \in \mathcal{P}\left(u_{1}, \ldots, u_{N}\right)$ define $\Phi_{P}: \operatorname{Int}(P) \rightarrow \mathbb{R}$ by

$$
\begin{aligned}
\Phi_{P}(\xi) & =\int_{S^{n-1}} \log (h(P, u)-\xi \cdot u) d \mu(u) \\
& =\sum_{k=1}^{N} \gamma_{k} \log \left(h\left(P, u_{k}\right)-\xi \cdot u_{k}\right),
\end{aligned}
$$

where Int $(P)$ is the interior of $P$.

In this section, we study the following extremal problem:

$$
\inf \left\{\max _{\xi \in \operatorname{Int}(Q)} \Phi_{Q}(\xi): Q \in \mathcal{P}\left(u_{1}, \ldots, u_{N}\right) \text { and } V(Q)=|\mu|\right\}
$$

where $|\mu|=\sum_{k=1}^{N} \gamma_{k}$.

We will prove that the solution of problem (3.2) solves the corresponding logarithmic Minkowski problem.

For the case where $u_{1}, \ldots, u_{N}$ are in general position and $Q \in \mathcal{P}_{N}\left(u_{1}, \ldots, u_{N}\right)$, problem (3.2) was studied in 69. The results and proofs in this section are similar to [69]. However, for convenience of the readers, we give detailed proofs for these results.

Lemma 3.1. Suppose $\mu=\sum_{k=1}^{N} \gamma_{k} \delta_{u_{k}}$ is a discrete measure on $S^{n-1}$ that is not concentrated on a closed hemisphere, and $P \in \mathcal{P}\left(u_{1}, \ldots, u_{N}\right)$, then there exists a unique point $\xi(P) \in \operatorname{Int}(P)$ such that

$$
\Phi_{P}(\xi(P))=\max _{\xi \in \operatorname{Int}(P)} \Phi_{P}(\xi) .
$$

Proof. Let $0<\lambda<1$ and $\xi_{1}, \xi_{2} \in \operatorname{Int}(P)$. From the concavity of the logarithmic function,

$$
\begin{aligned}
\lambda \Phi_{P}\left(\xi_{1}\right)+(1-\lambda) \Phi_{P}\left(\xi_{2}\right)= & \lambda \int_{S^{n-1}} \log \left(h(P, u)-\xi_{1} \cdot u\right) d \mu(u) \\
& \quad+(1-\lambda) \int_{S^{n-1}} \log \left(h(P, u)-\xi_{2} \cdot u\right) d \mu(u) \\
= & \sum_{k=1}^{N} \gamma_{k}\left[\lambda \log \left(h\left(P, u_{k}\right)-\xi_{1} \cdot u_{k}\right)+(1-\lambda) \log \left(h\left(P, u_{k}\right)-\xi_{2} \cdot u_{k}\right)\right] \\
\leq & \sum_{k=1}^{N} \gamma_{k} \log \left[h\left(P, u_{k}\right)-\left(\lambda \xi_{1}+(1-\lambda) \xi_{2}\right) \cdot u_{k}\right] \\
= & \Phi_{P}\left(\lambda \xi_{1}+(1-\lambda) \xi_{2}\right),
\end{aligned}
$$

with equality if and only if $\xi_{1} \cdot u_{k}=\xi_{2} \cdot u_{k}$ for all $k=1, \ldots, N$. Since the unit vectors $u_{1}, \ldots, u_{N}$ are not concentrated on a closed hemisphere, $\mathbb{R}^{n}=\operatorname{lin}\left\{u_{1}, \ldots, u_{N}\right\}$. Thus, $\xi_{1}=\xi_{2}$. Therefore, $\Phi_{P}$ is strictly concave on Int $(P)$.

Since $P \in \mathcal{P}\left(u_{1}, \ldots, u_{N}\right)$, for any $x \in \partial P$, there exists some $i_{0} \in\{1, \ldots, N\}$ such that

$$
h\left(P, u_{i_{0}}\right)=x \cdot u_{i_{0}} .
$$


Thus, $\Phi_{P}(\xi) \rightarrow-\infty$ whenever $\xi \in \operatorname{Int}(P)$ and $\xi \rightarrow x$. Therefore, there exists a unique interior point $\xi(P)$ of $P$ such that

$$
\Phi_{P}(\xi(P))=\max _{\xi \in \operatorname{Int}(P)} \Phi_{P}(\xi)
$$

Obviously, for $\lambda>0$ and $P \in \mathcal{P}\left(u_{1}, \ldots, u_{N}\right)$,

$$
\xi(\lambda P)=\lambda \xi(P)
$$

and if $P_{i} \in \mathcal{P}\left(u_{1}, \ldots, u_{N}\right)$ and $P_{i}$ converges to a polytope $P$, then $P \in \mathcal{P}\left(u_{1}, \ldots, u_{N}\right)$.

For the case where $u_{1}, \ldots, u_{N}$ are in general position, the following lemma was proved in 69.

Lemma 3.2. Suppose $\mu=\sum_{k=1}^{N} \gamma_{k} \delta_{u_{k}}$ is a discrete measure on $S^{n-1}$ that is not concentrated on a closed hemisphere, $P_{i} \in \mathcal{P}\left(u_{1}, \ldots, u_{N}\right)$ and $P_{i}$ converges to a polytope $P$, then $\lim _{i \rightarrow \infty} \xi\left(P_{i}\right)=\xi(P)$ and

$$
\lim _{i \rightarrow \infty} \Phi_{P_{i}}\left(\xi\left(P_{i}\right)\right)=\Phi_{P}(\xi(P))
$$

Proof. Since $\xi(P) \in \operatorname{Int}(P)$ by Lemma 3.1, we have

$$
\liminf _{i \rightarrow \infty} \Phi_{P_{i}}\left(\xi\left(P_{i}\right)\right) \geq \liminf _{i \rightarrow \infty} \Phi_{P_{i}}(\xi(P))=\Phi_{P}(\xi(P))
$$

Let $z$ be any accumulation point of the sequence $\left\{\xi\left(P_{i}\right)\right\}$; namely, the limit of a subsequence $\left\{\xi\left(P_{i^{\prime}}\right)\right\}$. Since $\Phi_{P_{i}}\left(\xi\left(P_{i}\right)\right)$ is bounded from below, and $h\left(P, u_{k}\right)-\xi\left(P_{i}\right) \cdot u_{k}$ is bounded from above for $k=1, \ldots, N$, it follows that

$$
\liminf _{i \rightarrow \infty}\left(h\left(P, u_{k}\right)-\xi\left(P_{i}\right) \cdot u_{k}\right)=\liminf _{i \rightarrow \infty}\left(h\left(P_{i}, u_{k}\right)-\xi\left(P_{i}\right) \cdot u_{k}\right)>0
$$

for $k=1, \ldots, N$, and hence $z \in \operatorname{Int}(P)$. We deduce that

$$
\Phi_{P}(z)=\lim _{i^{\prime} \rightarrow \infty} \Phi_{P}\left(\xi\left(P_{i^{\prime}}\right)\right)=\lim _{i^{\prime} \rightarrow \infty} \Phi_{P_{i^{\prime}}}\left(\xi\left(P_{i^{\prime}}\right)\right) \geq \liminf _{i \rightarrow \infty} \Phi_{P_{i}}\left(\xi\left(P_{i}\right)\right) \geq \Phi_{P}(\xi(P)) .
$$

Therefore Lemma 3.1 yields $z=\xi(P)$.

The following lemma will be needed, as well.

Lemma 3.3. Suppose $\mu=\sum_{k=1}^{N} \gamma_{k} \delta_{u_{k}}$ is a discrete measure on $S^{n-1}$ that is not concentrated on a closed hemisphere, $P \in \mathcal{P}\left(u_{1}, \ldots, u_{N}\right)$, then

$$
\sum_{k=1}^{N} \gamma_{k} \frac{u_{k}}{h\left(P, u_{k}\right)-\xi(P) \cdot u_{k}}=0
$$

Proof. We may assume that $\xi(P)$ is the origin because for $x, \xi \in \operatorname{Int} P$, we have $\Phi_{P-x}(\xi-x)=$ $\Phi_{P}(\xi)$. Since $\Phi_{P}(\xi)$ attains its maximum at the origin that is an interior point of $P$, differentiation gives the desired equation.

Lemma 3.4. Suppose $\mu=\sum_{k=1}^{N} \gamma_{k} \delta_{u_{k}}$ is a discrete measure on $S^{n-1}$ that is not concentrated on a closed hemisphere, and there exists a $P \in \mathcal{P}_{N}\left(u_{1}, \ldots, u_{N}\right)$ with $\xi(P)=0, V(P)=|\mu|$ such that

$$
\Phi_{P}(0)=\inf \left\{\max _{\xi \in \operatorname{Int}(Q)} \Phi_{Q}(\xi): Q \in \mathcal{P}\left(u_{1}, \ldots, u_{N}\right) \text { and } V(Q)=|\mu|\right\} \text {. }
$$

Then,

$$
V_{P}=\sum_{k=1}^{N} \gamma_{k} \delta_{u_{k}}
$$


Proof. According to Equation (3.3), it is sufficient to establish the lemma under the assumption that $|\mu|=1$.

From the conditions, there exists a polytope $P \in \mathcal{P}_{N}\left(u_{1}, \ldots, u_{N}\right)$ with $\xi(P)$ is the origin and $V(P)=1$ such that

$$
\Phi_{P}(o)=\inf \left\{\max _{\xi \in \operatorname{Int}(Q)} \Phi_{Q}(\xi): Q \in \mathcal{P}\left(u_{1}, \ldots, u_{N}\right) \text { and } V(Q)=1\right\} .
$$

For $\tau_{1}, \ldots, \tau_{N} \in \mathbb{R}$, choose $|t|$ small enough so that the polytope

$$
P_{t}=\bigcap_{i=1}^{N}\left\{x: x \cdot u_{i} \leq h\left(P, u_{i}\right)+t \tau_{i}\right\} \in \mathcal{P}_{N}\left(u_{1}, \ldots, u_{N}\right) .
$$

In particular, $h\left(P_{t}, u_{i}\right)=h\left(P, u_{i}\right)+t \tau_{i}$ for $i=1, \ldots, n$, and Lemma 7.5.3 in Schneider [61] yields that

$$
\frac{\partial V\left(P_{t}\right)}{\partial t}=\sum_{i=1}^{N} \tau_{i}\left|F\left(P_{t}, u_{i}\right)\right|
$$

Let $\lambda(t)=V\left(P_{t}\right)^{-\frac{1}{n}}$. Then $\lambda(t) P_{t} \in \mathcal{P}_{N}\left(u_{1}, \ldots, u_{N}\right), V\left(\lambda(t) P_{t}\right)=1, \lambda(t)$ is $C^{1}$ and

$$
\lambda^{\prime}(0)=-\frac{1}{n} \sum_{i=1}^{N} \tau_{i}\left|F\left(P, u_{i}\right)\right|
$$

Define $\xi(t):=\xi\left(\lambda(t) P_{t}\right)$, and

$$
\begin{aligned}
\Phi(t) & :=\max _{\xi \in \lambda(t) P_{t}} \int_{S^{n-1}} \log \left(h\left(\lambda(t) P_{t}, u\right)-\xi \cdot u\right) d \mu(u) \\
& =\sum_{k=1}^{N} \gamma_{k} \log \left(\lambda(t) h\left(P_{t}, u_{k}\right)-\xi(t) \cdot u_{k}\right) .
\end{aligned}
$$

It follows from Lemma 3.3, that

$$
\sum_{k=1}^{N} \gamma_{k} \frac{u_{k, i}}{\lambda(t) h\left(P_{t}, u_{k}\right)-\xi(t) \cdot u_{k}}=0
$$

for $i=1, \ldots, n$, where $u_{k}=\left(u_{k, 1}, \ldots, u_{k, n}\right)^{T}$. In addition, since $\xi(P)$ is the origin, we have

$$
\sum_{k=1}^{N} \gamma_{k} \frac{u_{k}}{h\left(P, u_{k}\right)}=0
$$

Let $F=\left(F_{1}, \ldots, F_{n}\right)$ be a function from a small neighbourhood of the origin in $\mathbb{R}^{n+1}$ to $\mathbb{R}^{n}$ such that

$$
F_{i}\left(t, \xi_{1}, \ldots, \xi_{n}\right)=\sum_{k=1}^{N} \gamma_{k} \frac{u_{k, i}}{\lambda(t) h\left(P_{t}, u_{k}\right)-\left(\xi_{1} u_{k, 1}+\ldots+\xi_{n} u_{k, n}\right)}
$$

for $i=1, \ldots, n$. Then,

$$
\begin{aligned}
& \left.\frac{\partial F_{i}}{\partial t}\right|_{\left(t, \xi_{1}, \ldots, \xi_{n}\right)}=\sum_{k=1}^{N} \gamma_{k} \frac{-u_{k, i}\left(\lambda^{\prime}(t) h\left(P_{t}, u_{k}\right)+\lambda(t) \tau_{k}\right)}{\left[\lambda(t) h\left(P_{t}, u_{k}\right)-\left(\xi_{1} u_{k, 1}+\ldots+\xi_{n} u_{k, n}\right)\right]^{2}} \\
& \left.\frac{\partial F_{i}}{\partial \xi_{j}}\right|_{\left(t, \xi_{1}, \ldots, \xi_{n}\right)}=\sum_{k=1}^{N} \gamma_{k} \frac{u_{k, i} u_{k, j}}{\left[\lambda(t) h\left(P_{t}, u_{k}\right)-\left(\xi_{1} u_{k, 1}+\ldots+\xi_{n} u_{k, n}\right)\right]^{2}}
\end{aligned}
$$


are continuous on a small neighborhood of $(0,0, \ldots, 0)$ with

$$
\left(\left.\frac{\partial F}{\partial \xi}\right|_{(0, \ldots, 0)}\right)_{n \times n}=\sum_{k=1}^{N} \frac{\gamma_{k}}{h\left(P, u_{k}\right)^{2}} u_{k} u_{k}^{T},
$$

where $u_{k} u_{k}^{T}$ is an $n \times n$ matrix. Since the unit vectors $u_{1}, \ldots, u_{N}$ are not concentrated on a closed hemisphere, $\mathbb{R}^{n}=\operatorname{lin}\left\{u_{1}, \ldots, u_{N}\right\}$. Thus, for any $x \in \mathbb{R}^{n}$ with $x \neq 0$, there exists a $u_{i_{0}} \in\left\{u_{1}, \ldots, u_{N}\right\}$ such that $u_{i_{0}} \cdot x \neq 0$. Then,

$$
\begin{aligned}
x^{T}\left(\sum_{k=1}^{N} \frac{\gamma_{k}}{h\left(P, u_{k}\right)^{2}} u_{k} u_{k}^{T}\right) x & =\sum_{k=1}^{N} \frac{\gamma_{k}}{h\left(P, u_{k}\right)^{2}}\left(x \cdot u_{k}\right)^{2} \\
& \geq \frac{\gamma_{i_{0}}}{h\left(P, u_{i_{0}}\right)^{2}}\left(x \cdot u_{i_{0}}\right)^{2}>0 .
\end{aligned}
$$

Therefore, $\left(\left.\frac{\partial F}{\partial \xi}\right|_{(0, \ldots, 0)}\right)$ is positive definite. By this, the fact that $F_{i}(0, \ldots, 0)=0$ for $i=1, \ldots, n$, the fact that $\frac{\partial F_{i}}{\partial \xi_{j}}$ is continuous on a neighborhood of $(0,0, \ldots, 0)$ for all $1 \leq i, j \leq n$ and the implicit function theorem, we have

$$
\xi^{\prime}(0)=\left(\xi_{1}^{\prime}(0), \ldots, \xi_{n}^{\prime}(0)\right)
$$

exists.

From the fact that $\Phi(0)$ is a minimizer of $\Phi(t)$ (in Equation (3.6)), Equation (3.5), the fact that $\sum_{k=1}^{N} \gamma_{k}=1$ and Equation (3.8), we have

$$
\begin{aligned}
0 & =\Phi^{\prime}(0) \\
& =\sum_{k=1}^{N} \gamma_{k} \frac{\lambda^{\prime}(0) h\left(P, u_{k}\right)+\left.\lambda(0) \frac{d h\left(P_{t}, u_{k}\right)}{d t}\right|_{t=0}-\xi^{\prime}(0) \cdot u_{k}}{h\left(P, u_{k}\right)} \\
& =\sum_{k=1}^{N} \gamma_{k} \frac{-\frac{1}{n}\left(\sum_{i=1}^{N} \tau_{i}\left|F\left(P, u_{i}\right)\right|\right) h\left(P, u_{k}\right)+\tau_{k}-\xi^{\prime}(0) \cdot u_{k}}{h\left(P, u_{k}\right)} \\
& =-\sum_{i=1}^{N} \frac{\left|F\left(P, u_{i}\right)\right| \tau_{i}}{n}+\sum_{k=1}^{N} \frac{\gamma_{k} \tau_{k}}{h\left(P, u_{k}\right)}-\xi^{\prime}(0) \cdot\left[\sum_{k=1}^{N} \gamma_{k} \frac{u_{k}}{h\left(P, u_{k}\right)}\right] \\
& =\sum_{k=1}^{N}\left(\frac{\gamma_{k}}{h\left(P, u_{k}\right)}-\frac{\left|F\left(P, u_{k}\right)\right|}{n}\right) \tau_{k} .
\end{aligned}
$$

Since $\tau_{1}, \ldots, \tau_{N}$ are arbitrary, we deduce that $\gamma_{k}=\frac{1}{n} h\left(P, u_{k}\right)\left|F\left(P, u_{k}\right)\right|$ for $k=1, \ldots, N$.

\section{Existence of a solution of the extremal problem}

In this section, we prove Lemma 4.7 about the existence of a solution of problem (3.2) for the case where the discrete measure is not concentrated on any closed hemisphere of $S^{n-1}$ and satisfies the strict essential subspace concentration inequality. Having the results of the previous section, the essential new ingredient is the following statement (see Lemma 4.5).

If $\mu$ is a discrete measure on $S^{n-1}$ that is not concentrated on any closed hemisphere of $S^{n-1}$ and satisfies the strict essential subspace concentration inequality, and $\left\{P_{m}\right\}$ is a sequence of polytopes of unit volume such that the set of outer unit normals of $P_{m}$ is a subset of the support of $\mu$, and $\lim _{m \rightarrow \infty} d\left(P_{m}\right)=\infty$ then

$$
\lim _{m \rightarrow \infty} \Phi_{P_{m}}\left(\xi\left(P_{m}\right)\right)=\infty
$$


It is equivalent to prove that any subsequence of $\left\{P_{m}\right\}$ has some subsequence $\left\{P_{m^{\prime}}\right\}$ such that $\lim _{m \rightarrow \infty} \Phi_{P_{m^{\prime}}}\left(\xi\left(P_{m^{\prime}}\right)\right)=\infty$.

To indicate the idea, we sketch the argument for $n=2$. Let $\operatorname{supp} \mu=\left\{u_{1}, \ldots, u_{N}\right\}$, and let $w_{m}=\min \left\{h_{P_{m}}(u)+h_{P_{m}}(-u): u \in S^{1}\right\}$ be the minimal width of $P_{m}$. Since $\lim _{m \rightarrow \infty} d\left(P_{m}\right)=\infty$ and $V\left(P_{m}\right)=1$, we have $\lim _{m \rightarrow \infty} w_{m}=0$. As $P_{m}$ is a polygon, we may assume that $w_{m}=$ $h_{P_{m}}\left(u_{1}\right)+h_{P_{m}}\left(-u_{1}\right)$ possibly after taking a subsequence and reindexing. If the angle of $u_{1}$ and $u_{i}$ is $\alpha_{i} \in(0, \pi)$ then $V_{1}\left(F\left(P_{m}, u_{i}\right)\right) \leq w_{m} / \sin \alpha_{i}$, thus $\lim _{m \rightarrow \infty} d\left(P_{m}\right)=\infty$ implies that $-u_{1} \in \operatorname{supp} \mu$ for large $m$, say $u_{2}=-u_{1}$. Let $v \in S^{1}$ be orthogonal to $u_{1}$, and let $\gamma_{i}=\mu\left(\left\{u_{i}\right\}\right)$ for $i=1, \ldots, N$. We may translate $P_{m}$ in a way such that $o \in \operatorname{Int} P_{m}$ in a way such that $h_{P_{m}}\left(u_{1}\right)=h_{P_{m}}\left(u_{2}\right)=w_{m} / 2$, and $h_{P_{m}}(v)=h_{P_{m}}(-v)$ hold for large $m$. Thus $V\left(P_{m}\right)=1$ yields the existence of a constant $c_{1}>0$ such that $h_{P_{m}}\left(u_{i}\right)>c_{1} / w_{m}$ for $i=3, \ldots, N$. Now lin $u_{1}$ is an essential subspace with respect to $\mu$, and hence $\gamma_{1}+\gamma_{2}<\gamma_{3}+\ldots+\gamma_{N}$ according to the strict essential subspace concentration inequality. Therefore writing $c_{2}=\min \left\{2, c_{1}\right\}$, we have

$$
\begin{aligned}
\liminf _{m \rightarrow \infty} \exp \left(\Phi_{P_{m}}\left(\xi\left(P_{m}\right)\right)\right) & \geq \liminf _{m \rightarrow \infty} \exp \left(\Phi_{P_{m}}(o)\right)=\liminf _{m \rightarrow \infty} \prod_{i=1}^{N} h_{P_{m}}\left(u_{i}\right)^{\gamma_{i}} \\
& \geq \lim _{m \rightarrow \infty}\left(\frac{w_{m}}{2}\right)^{\gamma_{1}+\gamma_{2}}\left(\frac{c_{1}}{w_{m}}\right)^{\gamma_{3}+\ldots+\gamma_{N}} \geq \lim _{m \rightarrow \infty}\left(\frac{c_{2}}{w_{m}}\right)^{\gamma_{3}+\ldots+\gamma_{N}-\gamma_{1}-\gamma_{2}}=\infty
\end{aligned}
$$

In the higher dimensional case, the idea is the very same. Only instead of one essential linear subspace like in the planar case, we will find essential subspaces $X_{0} \subset \ldots \subset X_{q-1}$ in a way such that for $j=0, \ldots, q-1,\left.P_{m}\right|_{X_{j}^{\perp}}$ is "much larger" than $\left.P_{m}\right|_{X_{j}}$ for large $m$ after taking suitable subsequence. This is achieved in the preparatory statements Lemmas 4.1 to 4.4 .

Given $N$ sequences, the first two observations will help to do book keeping of how the limits of the sequences compare.

Lemma 4.1. Let $\left\{h_{1 j}\right\}_{j=1}^{\infty}, \ldots,\left\{h_{N j}\right\}_{j=1}^{\infty}$ be $N(N \geq 2)$ sequences of real numbers. Then, there exists a subsequence, $\left\{j_{n}\right\}_{n=1}^{\infty}$, of $\mathbb{N}$ and a rearrangement, $i_{1}, \ldots, i_{N}$, of $1, \ldots, N$ such that

$$
h_{i_{1} j_{n}} \leq h_{i_{2} j_{n}} \leq \ldots \leq h_{i_{N} j_{n}}
$$

for all $n \in \mathbb{N}$.

Proof. We prove it by induction on $N$. We first prove the case for $N=2$. For $j \in \mathbb{N}$, consider the sequence

$$
h_{j}=\max \left\{h_{1 j}, h_{2 j}\right\} .
$$

Since $\left\{h_{j}\right\}_{j=1}^{\infty}$ is an infinite sequence and $h_{j}$ either equals to $h_{1 j}$ or equals to $h_{2 j}$ for all $j \in \mathbb{N}$, there exists an $i_{2} \in\{1,2\}$ and a subsequence, $\left\{j_{n}\right\}_{n=1}^{\infty}$, of $\mathbb{N}$ such that

$$
h_{j_{n}}=h_{i_{2} j_{n}}
$$

for all $n \in \mathbb{N}$. Let $i_{1} \in\{1,2\}$ with $i_{1} \neq i_{2}$. Then,

$$
h_{i_{1} j_{n}} \leq h_{i_{2} j_{n}}
$$

for all $n \in \mathbb{N}$.

Suppose the lemma is true for $N=k$ (with $k \geq 2$ ), we next prove that the lemma is true for $N=k+1$. For $j \in \mathbb{N}$, consider the sequence

$$
h_{j}=\max \left\{h_{1 j}, h_{2 j}, \ldots, h_{k+1 j}\right\} .
$$

Since $\left\{h_{j}\right\}_{j=1}^{\infty}$ is an infinite sequence and $h_{j}$ equals one of $h_{1 j}, h_{2 j}, \ldots, h_{k+1 j}$ for all $j \in \mathbb{N}$, there exists an $i_{k+1} \in\{1,2, \ldots, k+1\}$ and a subsequence, $\left\{j_{n}\right\}_{n=1}^{\infty}$, of $\mathbb{N}$ such that

$$
h_{j_{n}}=h_{i_{k+1} j_{n}}
$$


for all $n \in \mathbb{N}$.

Consider the sequences $\left\{h_{i j_{n}}\right\}_{n=1}^{\infty}\left(1 \leq i \leq k+1\right.$ with $\left.i \neq i_{k+1}\right)$. By the inductive hypothesis, there exists a subsequence, $j_{n_{l}}$, of $j_{n}$ and a rearrangement, $i_{1}, \ldots, i_{k}$, of $1, \ldots, \widehat{i_{k+1}}, \ldots, k+1$ such that

$$
h_{i_{1} j_{n_{l}}} \leq h_{i_{2} j_{n_{l}}} \leq \ldots \leq h_{i_{k} j_{n_{l}}}
$$

for all $l \in \mathbb{N}$. By this and the fact that $h_{j_{n_{l}}}=h_{i_{k+1} j_{n_{l}}}$ for all $l \in \mathbb{N}$, we have

$$
h_{i_{1} j_{n_{l}}} \leq h_{i_{2} j_{n_{l}}} \leq \ldots \leq h_{i_{k} j_{n_{l}}} \leq h_{i_{k+1} j_{n_{l}}}
$$

for all $l \in \mathbb{N}$.

Lemma 4.2. Let $\left\{h_{1 j}\right\}_{j=1}^{\infty}, \ldots,\left\{h_{N j}\right\}_{j=1}^{\infty}$ be $N(N \geq 2)$ sequences of real numbers with

$$
h_{1 j} \leq h_{2 j} \leq \ldots \leq h_{N j}
$$

for all $j \in \mathbb{N}, \lim _{j \rightarrow \infty} h_{1 j}=0$ and $\lim _{j \rightarrow \infty} h_{N j}=\infty$. Then, there exist $q \geq 1$,

$$
1=\alpha_{0}<\alpha_{1}<\ldots<\alpha_{q} \leq N<N+1=\alpha_{q+1}
$$

and a subsequence, $\left\{j_{n}\right\}_{n=1}^{\infty}$, of $\mathbb{N}$ such that if $i=1, \ldots, q$, then

$$
\lim _{n \rightarrow \infty} \frac{h_{\alpha_{i} j_{n}}}{h_{\alpha_{i-1} j_{n}}}=\infty
$$

if $i=0, \ldots, q$, and $\alpha_{i} \leq k \leq \alpha_{i+1}-1$, then

$$
\lim _{n \rightarrow \infty} \frac{h_{k j_{n}}}{h_{\alpha_{i} j_{n}}}
$$

exists and equals to a positive number.

Proof. Let $\alpha_{0}=1$. By conditions,

$$
\frac{h_{1 j}}{h_{1 j}} \leq \frac{h_{2 j}}{h_{1 j}} \leq \ldots \leq \frac{h_{N j}}{h_{1 j}}
$$

$\varlimsup_{j \rightarrow \infty} \frac{h_{i j}}{h_{1 j}}$ either exists (equals to a positive number) or goes to $\infty$, and $\varlimsup_{j \rightarrow \infty} \frac{h_{N j}}{h_{1 j}}=\infty$. Thus, there exists an $\alpha_{1}\left(1<\alpha_{1} \leq N\right)$ such that for $1 \leq i \leq \alpha_{1}-1$,

$$
\varlimsup_{j \rightarrow \infty} \frac{h_{i j}}{h_{1 j}}<\infty
$$

and

$$
\varlimsup_{j \rightarrow \infty} \frac{h_{\alpha_{1} j}}{h_{1 j}}=\infty .
$$

Hence, we can choose a subsequence, $\left\{j_{n}^{\prime}\right\}_{n=1}^{\infty}$, of $\mathbb{N}$ such that

$$
\lim _{n \rightarrow \infty} \frac{h_{\alpha_{1} j_{n}^{\prime}}}{h_{1 j_{n}^{\prime}}}=\infty
$$

and for $1 \leq i \leq \alpha_{1}-1$,

$$
\varlimsup_{n \rightarrow \infty} \frac{h_{i j_{n}^{\prime}}}{h_{1 j_{n}^{\prime}}} \leq \varlimsup_{j \rightarrow \infty} \frac{h_{i j}}{h_{1 j}}<\infty .
$$

By choosing $\alpha_{1}-2$ times subsequences of $j_{n}^{\prime}$, we can find a subsequence, $\left\{j_{n}^{\prime \prime}\right\}_{n=1}^{\infty}$, of $\left\{j_{n}^{\prime}\right\}_{n=1}^{\infty}$ such that

and for $1 \leq i \leq \alpha_{1}-1$,

$$
\lim _{n \rightarrow \infty} \frac{h_{\alpha_{1} j_{n}^{\prime \prime}}}{h_{1 j_{n}^{\prime \prime}}}=\infty
$$

$$
\lim _{n \rightarrow \infty} \frac{h_{i j_{n}^{\prime \prime}}}{h_{1 j_{n}^{\prime \prime}}}
$$


exists and equals to a positive number.

By repeating (at most $N-\alpha_{1}$ times) similar arguments for the sequences $\left\{h_{i j_{n}^{\prime \prime}}\right\}_{n=1}^{\infty}\left(\alpha_{1} \leq i \leq N\right)$, we can find $q \geq 1$,

$$
1=\alpha_{0}<\alpha_{1}<\ldots<\alpha_{q} \leq N<N+1=\alpha_{q+1}
$$

and a subset, $\left\{j_{n}\right\}_{n=1}^{\infty}$, of $\mathbb{N}$ that satisfy the conditions in the lemma.

The following lemma compares positive hull and linear hull.

Lemma 4.3. Suppose $u_{1}, \ldots, u_{l} \in S^{d-1}(d \geq 2), \mathbb{R}^{d}=\operatorname{lin}\left\{u_{1}, \ldots, u_{l}\right\}$, and $u_{1}, \ldots, u_{l}$ are not concentrated on a closed hemisphere of $S^{d-1}$, then

$$
\mathbb{R}^{d}=\operatorname{pos}\left\{u_{1}, \ldots, u_{l}\right\}
$$

Moreover, there exists $\lambda>0$ depending on $u_{1}, \ldots, u_{l}$ such that any $u \in S^{d-1}$ can be written in the form

$$
u=a_{i_{1}} u_{i_{1}}+\ldots+a_{i_{d}} u_{i_{d}}
$$

where $\left\{u_{i_{1}}, \ldots, u_{i_{d}}\right\} \subset\left\{u_{1}, \ldots, u_{l}\right\}$ and $0 \leq a_{i_{1}}, \ldots, a_{i_{d}} \leq \lambda$.

Proof. Let $Q$ be the convex hull of $\left\{u_{1}, \ldots, u_{l}\right\}$, which is a polytope. Since $u_{1}, \ldots, u_{l}$ are not concentrated on a closed hemisphere of $S^{d-1}$, the origin is an interior point of $Q$. In particular, $r B^{d} \subset Q$ for some $r>0$.

For $u \in S^{d-1}$, there exists some $t \geq r$ such that $t u \in \partial Q$. It follows that $t u \in F$ for some facet $F$ of $Q$. We deduce from the Charateodory theorem that there exists vertices $u_{i_{1}}, \ldots, u_{i_{d}}$ of $F$ that $t u$ lies in their convex hull. In other words,

$$
t u=\alpha_{i_{1}} u_{i_{1}}+\ldots+\alpha_{i_{d}} u_{i_{d}}
$$

where $\alpha_{i_{1}}, \ldots, \alpha_{i_{d}} \geq 0$ and $\alpha_{i_{1}}+\ldots+\alpha_{i_{d}}=1$. Therefore we choose $a_{i_{j}}=\alpha_{i_{j}} / t \leq 1 / r$ for $j=1, \ldots, d$, which in turn satisfy $u=a_{i_{1}} u_{i_{1}}+\ldots+a_{i_{d}} u_{i_{d}}$. In particular, we may take $\lambda=1 / r$.

The following lemma will be the last preparatory statement.

Lemma 4.4. Suppose $\mu$ is a discrete measure on $S^{n-1}$ that is not concentrated on any closed hemisphere of $S^{n-1}$ with $\operatorname{supp}(\mu)=\left\{u_{1}, \ldots, u_{N}\right\}$ and $\mu\left(u_{i}\right)=\gamma_{i}$ for $i=1, \ldots, N$. If $P_{m}$ is a sequence of polytopes with $V\left(P_{m}\right)=1, \xi\left(P_{m}\right)$ is the origin, the set of outer unit normals of $P_{m}$ is a subset of $\left\{u_{1}, \ldots, u_{N}\right\}, \lim _{m \rightarrow \infty} d\left(P_{m}\right)=\infty$ and

$$
h\left(P_{m}, u_{1}\right) \leq h\left(P_{m}, u_{2}\right) \leq \ldots \leq h\left(P_{m}, u_{N}\right)
$$

for all $m \in \mathbb{N}$. Then, there exist $q \geq 1$, and $1=\alpha_{0}<\alpha_{1}<\ldots<\alpha_{q} \leq N<N+1=\alpha_{q+1}$ such that if $j=1, \ldots, q$, then

$$
\lim _{m \rightarrow \infty} \frac{h\left(P_{m}, u_{\alpha_{j}}\right)}{h\left(P_{m}, u_{\alpha_{j-1}}\right)}=\infty,
$$

and if $j=0, \ldots, q$ and $\alpha_{j} \leq k \leq \alpha_{j+1}-1$, then

$$
\lim _{m \rightarrow \infty} \frac{h\left(P_{m}, u_{k}\right)}{h\left(P_{m}, u_{\alpha_{j}}\right)}=t_{k j}<\infty .
$$

Moreover, $X_{j}=\operatorname{pos}\left\{u_{1}, \ldots, u_{\alpha_{j+1}-1}\right\}$ are subspaces of $\mathbb{R}^{n}$ for all $0 \leq j \leq q$ and

$$
1 \leq \operatorname{dim}\left(X_{0}\right)<\operatorname{dim}\left(X_{1}\right)<\ldots<\operatorname{dim}\left(X_{q}\right)=n .
$$


Proof. By the conditions that $\lim _{m \rightarrow \infty} d\left(P_{m}\right)=\infty, V(K)=1$ and $h\left(P_{m}, u_{1}\right) \leq h\left(P_{m}, u_{2}\right) \leq \ldots \leq$ $h\left(P_{m}, u_{N}\right)$ for all $m \in \mathbb{N}$, we have,

$$
\lim _{m \rightarrow \infty} h\left(P_{m}, u_{1}\right)=0 \text { and } \lim _{m \rightarrow \infty} h\left(P_{m}, u_{N}\right)=\infty .
$$

From Lemma 4.2, we may assume that there exist $q \geq 1$, and

$$
1=\alpha_{0}<\alpha_{1}<\ldots<\alpha_{q} \leq N<N+1=\alpha_{q+1}
$$

that satisfy Equations (4.0a) and (4.0b).

For $j=0, \ldots, q-1$, we consider the cone

$$
\Sigma_{j}=\operatorname{pos}\left\{u_{1}, \ldots, u_{\alpha_{j+1}-1}\right\},
$$

and its negative polar

$$
\Sigma_{j}^{*}=\left\{v \in \mathbb{R}^{n}: v \cdot u_{i} \leq 0 \text { for all } i=1, \ldots, \alpha_{j+1}-1\right\} .
$$

Let $0 \leq j \leq q-1,1 \leq p \leq \alpha_{j+1}-1$ and $v \in \Sigma_{j}^{*} \cap S^{n-1}$. From the condition that $\xi\left(P_{m}\right)$ is the origin and Lemma 3.3 ,

$$
\sum_{i=1}^{N} \frac{\gamma_{i}\left(v \cdot u_{i}\right)}{h\left(P_{m}, u_{i}\right)}=0
$$

By this and the fact that $v \in \Sigma_{j}^{*} \cap S^{n-1}$,

$$
\begin{aligned}
0 & \geq \gamma_{p}\left(v \cdot u_{p}\right)=-\sum_{i \neq p} \frac{h\left(P_{m}, u_{p}\right)}{h\left(P_{m}, u_{i}\right)} \gamma_{i}\left(v \cdot u_{i}\right) \\
& \geq-\sum_{i \geq \alpha_{j+1}} \frac{h\left(P_{m}, u_{p}\right)}{h\left(P_{m}, u_{i}\right)} \gamma_{i}\left(v \cdot u_{i}\right) \\
& \geq-\sum_{i \geq \alpha_{j+1}} \frac{h\left(P_{m}, u_{p}\right)}{h\left(P_{m}, u_{i}\right)} \gamma_{i} .
\end{aligned}
$$

By this, (4.0a) and (4.0b), we have, $\gamma_{p}\left(v \cdot u_{p}\right)$ is no bigger than 0 , and no less than any negative number. Thus,

$$
v \cdot u_{p}=0
$$

for all $p=1, \ldots, \alpha_{j+1}-1$ and $v \in \Sigma_{j}^{*} \cap S^{n-1}$. Then, for any $u \in \operatorname{lin}\left\{u_{1}, \ldots, u_{\alpha_{j+1}-1}\right\}$ and $v \in \Sigma_{j}^{*}$, $u \cdot v=0$. Hence,

$$
\Sigma_{j}^{*} \cap \operatorname{lin}\left\{u_{1}, \ldots, u_{\alpha_{j+1}-1}\right\}=\{0\} .
$$

We claim that $\left\{u_{1}, \ldots, u_{\alpha_{j+1}-1}\right\}$ is not concentrated on a closed hemisphere of $S^{n-1} \cap \operatorname{lin}\left\{u_{1}, \ldots, u_{\alpha_{j+1}-1}\right\}$. Otherwise, there exists a vector $u_{0} \in \operatorname{lin}\left\{u_{1}, \ldots, u_{\alpha_{j+1}-1}\right\}$ such that $u_{0} \neq 0$ and $u_{0} \cdot u_{p} \leq 0$ for all $p=1, \ldots, \alpha_{j+1}-1$. This contradicts the fact that $\Sigma_{j}^{*} \cap \operatorname{lin}\left\{u_{1}, \ldots, u_{\alpha_{j+1}-1}\right\}=\{0\}$. Hence, $\left\{u_{1}, \ldots, u_{\alpha_{j+1}-1}\right\}$ is not concentrated on a closed hemisphere of $S^{n-1} \cap \operatorname{lin}\left\{u_{1}, \ldots, u_{\alpha_{j+1}-1}\right\}$. By Lemma 4.3,

$$
\operatorname{lin}\left\{u_{1}, \ldots, u_{\alpha_{j+1}-1}\right\}=\operatorname{pos}\left\{u_{1}, \ldots, u_{\alpha_{j+1}-1}\right\} .
$$

Let $X_{j}=\operatorname{pos}\left\{u_{1}, \ldots, u_{\alpha_{j+1}-1}\right\}, d_{j}=\operatorname{dim} X_{j}$ for $j=0, \ldots, q$, and $d_{-1}=0$. Obviously, $d_{0} \geq 1$ and $d_{q}=n$. We claim that $d_{0}<d_{1}<\ldots<d_{q}$. Otherwise, there exist $0 \leq k<l \leq q$ such that $d_{k}=d_{l}$, and thus $X_{k}=X_{l}$. We write $\lambda>0$ for the constant of Lemma 4.3 depending on $u_{1}, \ldots, u_{N}$. By Lemma 4.3, there exist $u_{i_{1}}, \ldots, u_{i_{d_{k}}} \in\left\{u_{1}, \ldots, u_{\alpha_{k+1}-1}\right\}$ and $0 \leq a_{i_{1}}, \ldots, a_{i_{d_{k}}} \leq \lambda$ such that

$$
u_{\alpha_{l}}=a_{i_{1}} u_{i_{1}}+\ldots+a_{i_{d_{k}}} u_{i_{d_{k}}} .
$$


Hence,

$$
\begin{aligned}
h\left(P_{m}, u_{\alpha_{l}}\right) & =h\left(P_{m}, a_{i_{1}} u_{i_{1}}+\ldots+a_{i_{d_{k}}} u_{i_{d_{k}}}\right) \\
& \leq a_{i_{1}} h\left(P_{m}, u_{i_{1}}\right)+\ldots+a_{i_{d_{k}}} h\left(P_{m}, u_{i_{d_{k}}}\right),
\end{aligned}
$$

for all $m \in \mathbb{N}$. But this contradicts (4.0a) and (4.0b). Therefore,

$$
1 \leq d_{0}<d_{1}<\ldots<d_{q}=n .
$$

Lemma 4.5. Suppose $\mu$ is a discrete measure on $S^{n-1}$ that is not concentrated on any closed hemisphere of $S^{n-1}$, and satisfies the strict essential subspace concentration inequality. If $P_{m}$ is a sequence of polytopes with $V\left(P_{m}\right)=1, \xi\left(P_{m}\right)$ is the origin, the set of outer unit normals of $P_{m}$ is a subset of the support of $\mu$ and $\lim _{m \rightarrow \infty} d\left(P_{m}\right)=\infty$, then

$$
\int_{S^{n-1}} \log h\left(P_{m}, u\right) d \mu(u)
$$

is not bounded from above.

Proof. Without loss of generality, we can suppose $|\mu|=1$. Let $\operatorname{supp}(\mu)=\left\{u_{1}, \ldots, u_{N}\right\}$, and $\mu\left(\left\{u_{i}\right\}\right)=\gamma_{i}, i=1, \ldots, N$. From Lemma 4.1, we may assume that

$$
h\left(P_{m}, u_{1}\right) \leq \ldots \leq h\left(P_{m}, u_{N}\right),
$$

for all $m \in \mathbb{N}$. Since $\lim _{m \rightarrow \infty} d\left(P_{m}\right)=\infty$ and $V(K)=1$,

$$
\lim _{m \rightarrow \infty} h\left(P_{m}, u_{1}\right)=0 \text { and } \lim _{m \rightarrow \infty} h\left(P_{m}, u_{N}\right)=\infty .
$$

By Lemma 4.4, there exist $q \geq 1$, and

$$
1=\alpha_{0}<\alpha_{1}<\ldots<\alpha_{q} \leq N<N+1=\alpha_{q+1}
$$

such that if $j=1, \ldots, q$, then

$$
\lim _{m \rightarrow \infty} \frac{h\left(P_{m}, u_{\alpha_{j}}\right)}{h\left(P_{m}, u_{\alpha_{j-1}}\right)}=\infty,
$$

and if $j=0, \ldots, q$ and $\alpha_{j} \leq k \leq \alpha_{j+1}-1$, then

$$
\lim _{m \rightarrow \infty} \frac{h\left(P_{m}, u_{k}\right)}{h\left(P_{m}, u_{\alpha_{j}}\right)}=t_{k, j}<\infty .
$$

Moreover, $X_{j}=\operatorname{pos}\left\{u_{1}, \ldots, u_{\alpha_{j+1}-1}\right\}$ are subspaces of $\mathbb{R}^{n}$ with respect to $\mu$ for all $0 \leq j \leq q$ with

$$
1 \leq d_{0}<d_{1}<\ldots<d_{q}=n,
$$

where $d_{j}=\operatorname{dim}\left(X_{j}\right)$. In particular, $X_{0}, \ldots, X_{q-1}$ are essential subspaces.

Let $\widetilde{X}_{0}=X_{0}$, and if $j=1, \ldots, q$, then let

$$
\tilde{X}_{j}=X_{j-1}^{\perp} \cap X_{j}
$$

From the definition of $X_{j}$ and $\tilde{X}_{j}$, we have, $\tilde{X}_{j_{1}} \perp \tilde{X}_{j_{2}}$ for $j_{1} \neq j_{2}$, $\operatorname{dim} \tilde{X}_{j}=d_{j}-d_{j-1}>0$ for $j=0, \ldots, q$, and $\mathbb{R}^{n}$ is a direct sum of $\tilde{X}_{0}, \ldots, \tilde{X}_{q}$.

Let $\lambda>0$ be the constant of Lemma 4.3 for $u_{1}, \ldots, u_{N}$. Suppose $0 \leq j \leq q$ and $u \in X_{j} \cap S^{n-1}$. By Lemma 4.3, there exists a subset, $\left\{u_{i_{1}}, \ldots, u_{i_{d_{j}}}\right\}$, of $\left\{u_{1}, \ldots, u_{\alpha_{j+1}-1}\right\}$ and $0 \leq a_{i_{1}}, \ldots, a_{i_{d_{j}}} \leq \lambda$ such that

$$
u=a_{i_{1}} u_{i_{1}}+\ldots+a_{i_{d_{j}}} u_{i_{d_{j}}} .
$$


Then,

$$
\begin{aligned}
h\left(P_{m}, u\right) & =h\left(P_{m}, a_{i_{1}} u_{i_{1}}+\ldots+a_{i_{d_{j}}} u_{i_{d_{j}}}\right) \\
& \leq a_{i_{1}} h\left(P_{m}, u_{i_{1}}\right)+\ldots+a_{i_{d_{j}}} h\left(P_{m}, u_{i_{d_{j}}}\right) .
\end{aligned}
$$

By this, (4.2a) and (4.2b), if $m$ is large, then

$$
h\left(P_{m}, u\right) \leq t_{j} h\left(P_{m}, u_{\alpha_{j}}\right) \text { for all } u \in X_{j} \cap S^{n-1}
$$

where $t_{j}=d_{j} \lambda\left(t_{\alpha_{j+1}-1, j}+1\right)>0$. Hence, for $j=0, \ldots, q$,

$$
\left.P_{m}\right|_{\tilde{X}_{j}} \subset t_{j} h\left(P_{m}, u_{\alpha_{j}}\right)\left(B^{n} \cap \tilde{X}_{j}\right) .
$$

By this and the fact that $\mathbb{R}^{n}$ is a direct sum of $\tilde{X}_{0}, \ldots, \tilde{X}_{q}$,

$$
P_{m} \subset \sum_{j=0}^{q} t_{j} h\left(P_{m}, u_{\alpha_{j}}\right)\left(B^{n} \cap \tilde{X}_{j}\right)
$$

where the summation is Minkowski sum. Let

$$
\omega=\max _{0 \leq j \leq q} t_{j} \kappa_{d_{j}-d_{j-1}}^{\frac{1}{d_{j}-d_{j-1}}}
$$

where $\kappa_{d_{j}-d_{j-1}}$ is the volume of the $\left(d_{j}-d_{j-1}\right)$-dimensional unit ball. Then, for $j=0, \ldots, q$

$$
V_{d_{j}-d_{j-1}}\left(t_{j} h\left(P_{m}, u_{\alpha_{j}}\right)\left(B^{n} \cap \tilde{X}_{j}\right)\right) \leq\left(\omega h\left(P_{m}, u_{\alpha_{j}}\right)\right)^{d_{j}-d_{j-1}}
$$

From this, the fact that $\mathbb{R}^{n}$ is a direct sum of $\tilde{X}_{0}, \ldots, \tilde{X}_{q}$, and Fubini's formula, we have

$$
\begin{aligned}
1 & =V\left(P_{m}\right) \\
& \leq V\left(\sum_{j=0}^{q} t_{j} h\left(P_{m}, u_{\alpha_{j}}\right)\left(B^{n} \cap \tilde{X}_{j}\right)\right) \\
& =\prod_{j=0}^{q} V_{d_{j}-d_{j-1}}\left(t_{j} h\left(P_{m}, u_{\alpha_{j}}\right)\left(B^{n} \cap \tilde{X}_{j}\right)\right) \\
& \leq \prod_{j=0}^{q}\left(\omega h\left(P_{m}, u_{\alpha_{j}}\right)\right)^{d_{j}-d_{j-1}} .
\end{aligned}
$$

It follows from $0=d_{-1}<d_{0}<\ldots<d_{q}=n$ that if $m$ is large, then

$$
\sum_{j=0}^{q}\left(\frac{d_{j}}{n}-\frac{d_{j-1}}{n}\right) \log h\left(P_{m}, u_{\alpha_{j}}\right) \geq-\log \omega .
$$

We rewrite the last inequality as

$$
\log h\left(P_{m}, u_{\alpha_{q}}\right) \geq-\sum_{j=0}^{q-1} \frac{d_{j}}{n} \log \frac{h\left(P_{m}, u_{\alpha_{j}}\right)}{h\left(P_{m}, u_{\alpha_{j+1}}\right)}-\log \omega .
$$

For $j=0, \ldots, q$, we set $\beta_{j}=\mu\left(X_{j} \cap S^{n-1}\right)=\sum_{i=1}^{\alpha_{j+1}-1} \gamma_{i}$, and $\beta_{-1}=0$. We deduce from the facts that $X_{j}$ is an essential subspace with $d_{j}=\operatorname{dim}\left(X_{j}\right)$, and from the condition that $\mu$ satisfies the strict essential subspace concentration condition that

$$
\beta_{j}<\frac{d_{j}}{n} \quad \text { for } 0 \leq j \leq q-1
$$


By the fact that $h\left(P_{m}, u_{1}\right) \leq h\left(P_{m}, u_{2}\right) \leq \ldots \leq h\left(P_{m}, u_{N}\right)$, the fact that $\beta_{q}=1$ and $(4.3)$,

$$
\begin{aligned}
\sum_{i=1}^{N} \gamma_{i} \log h\left(P_{m}, u_{i}\right) & =\sum_{i=1}^{\alpha_{1}-1} \gamma_{i} \log h\left(P_{m}, u_{i}\right)+\sum_{i=\alpha_{1}}^{\alpha_{2}-1} \gamma_{i} \log h\left(P_{m}, u_{i}\right)+\ldots+\sum_{i=\alpha_{q}}^{N} \gamma_{i} \log h\left(P_{m}, u_{i}\right) \\
& \geq \sum_{i=1}^{\alpha_{1}-1} \gamma_{i} \log h\left(P_{m}, u_{\alpha_{0}}\right)+\sum_{i=\alpha_{1}}^{\alpha_{2}-1} \gamma_{i} \log h\left(P_{m}, u_{\alpha_{1}}\right)+\ldots+\sum_{i=\alpha_{q}}^{N} \gamma_{i} \log h\left(P_{m}, u_{\alpha_{q}}\right) \\
& =\sum_{j=0}^{q}\left(\beta_{j}-\beta_{j-1}\right) \log h\left(P_{m}, u_{\alpha_{j}}\right) \\
& =\log h\left(P_{m}, u_{\alpha_{q}}\right)+\sum_{j=0}^{q-1} \beta_{j} \log \frac{h\left(P_{m}, u_{\alpha_{j}}\right)}{h\left(P_{m}, u_{\alpha_{j+1}}\right)} \\
& \geq-\log \omega+\sum_{j=0}^{q-1}\left(\beta_{j}-\frac{d_{j}}{n}\right) \log \frac{h\left(P_{m}, u_{\alpha_{j}}\right)}{h\left(P_{m}, u_{\alpha_{j+1}}\right)}
\end{aligned}
$$

It follows from (4.1), (4.2a), (4.4) that for $j=0, \ldots, q-1$,

$$
\lim _{m \rightarrow \infty}\left(\beta_{j}-\frac{d_{j}}{n}\right) \log \frac{h\left(P_{m}, u_{\alpha_{j}}\right)}{h\left(P_{m}, u_{\alpha_{j+1}}\right)}=\infty .
$$

Therefore,

$$
\lim _{m \rightarrow \infty} \sum_{i=1}^{N} \gamma_{i} \log h\left(P_{m}, u_{i}\right)=\infty
$$

The following lemma will be needed (see, [71], Lemma 3.5).

Lemma 4.6. If $P$ is a polytope in $\mathbb{R}^{n}$ and $v_{0} \in S^{n-1}$ with $V_{n-1}\left(F\left(P, v_{0}\right)\right)=0$, then there exists a $\delta_{0}>0$ such that for $0 \leq \delta<\delta_{0}$

$$
V\left(P \cap\left\{x: x \cdot v_{0} \geq h\left(P, v_{0}\right)-\delta\right\}\right)=c_{n} \delta^{n}+\ldots+c_{2} \delta^{2},
$$

where $c_{n}, \ldots, c_{2}$ are constants that depend on $P$ and $v_{0}$.

Now, we have prepared enough to prove the main result of this section.

Lemma 4.7. Suppose the discrete measure $\mu=\sum_{k=1}^{N} \gamma_{k} \delta_{u_{i}}$ is not concentrated on a closed hemisphere. If $\mu$ satisfies the strict essential subspace concentration inequality, then there exists a $P \in \mathcal{P}_{N}\left(u_{1}, \ldots, u_{N}\right)$ such that $\xi(P)=0, V(P)=|\mu|$ and

$$
\Phi_{P}(0)=\inf \left\{\max _{\xi \in \operatorname{Int}(Q)} \Phi_{Q}(\xi): Q \in \mathcal{P}\left(u_{1}, \ldots, u_{N}\right) \text { and } V(Q)=|\mu|\right\},
$$

where $\Phi_{Q}(\xi)=\int_{S^{n-1}} \log (h(Q, u)-\xi \cdot u) d \mu(u)$.

Proof. It is easily seen that it is sufficient to establish the lemma under the assumption that $|\mu|=1$.

Obviously, for $P, Q \in \mathcal{P}\left(u_{1}, \ldots, u_{N}\right)$, if there exists an $x \in \mathbb{R}^{n}$ such that $P=Q+x$, then

$$
\Phi_{P}(\xi(P))=\Phi_{Q}(\xi(Q))
$$

Thus, we can choose a sequence $P_{i} \in \mathcal{P}\left(u_{1}, \ldots, u_{N}\right)$ with $\xi\left(P_{i}\right)=0$ and $V\left(P_{i}\right)=1$ such that $\Phi_{P_{i}}(0)$ converges to

$$
\inf \left\{\max _{\xi \in \operatorname{Int}(Q)} \Phi_{Q}(\xi): Q \in \mathcal{P}\left(u_{1}, \ldots, u_{N}\right) \text { and } V(Q)=1\right\} \text {. }
$$


Choose a fixed $P_{0} \in \mathcal{P}\left(u_{1}, \ldots, u_{N}\right)$ with $V\left(P_{0}\right)=1$, then

$$
\inf \left\{\max _{\xi \in \operatorname{Int}(Q)} \Phi_{Q}(\xi): Q \in \mathcal{P}\left(u_{1}, \ldots, u_{N}\right) \text { and } V(Q)=1\right\} \leq \Phi_{P_{0}}\left(\xi\left(P_{0}\right)\right) \text {. }
$$

We claim that $P_{i}$ is bounded. Otherwise, from Lemma 4.5, $\Phi_{P_{i}}\left(\xi\left(P_{i}\right)\right)$ is not bounded from above. This contradicts the previous inequality. Therefore, $P_{i}$ is bounded.

From Lemma 3.2 and the Blaschke selection theorem, there exists a subsequence of $P_{i}$ that converges to a polytope $P$ such that $P \in \mathcal{P}\left(u_{1}, \ldots, u_{N}\right), V(P)=1, \xi(P)=0$ and

$$
\Phi_{P}(0)=\inf \left\{\max _{\xi \in \operatorname{Int}(Q)} \Phi_{Q}(\xi): Q \in \mathcal{P}\left(u_{1}, \ldots, u_{N}\right) \text { and } V(Q)=1\right\} .
$$

We next prove that $F\left(P, u_{i}\right)$ are facets for all $i=1, \ldots, N$. Otherwise, there exists an $i_{0} \in$ $\{1, \ldots, N\}$ such that

$$
F\left(P, u_{i_{0}}\right)
$$

is not a facet of $P$.

Choose $\delta>0$ small enough so that the polytope

$$
P_{\delta}=P \cap\left\{x: x \cdot u_{i_{0}} \leq h\left(P, u_{i_{0}}\right)-\delta\right\} \in \mathcal{P}\left(u_{1}, \ldots, u_{N}\right),
$$

and (by Lemma 4.6)

$$
V\left(P_{\delta}\right)=1-\left(c_{n} \delta^{n}+\ldots+c_{2} \delta^{2}\right),
$$

where $c_{n}, \ldots, c_{2}$ are constants that depend on $P$ and direction $u_{i_{0}}$.

From Lemma 3.2, for any $\delta_{i} \rightarrow 0 \xi\left(P_{\delta_{i}}\right) \rightarrow 0$. We have,

$$
\lim _{\delta \rightarrow 0} \xi\left(P_{\delta}\right)=0 .
$$

Let $\delta$ be small enough so that $h\left(P, u_{k}\right)>\xi\left(P_{\delta}\right) \cdot u_{k}+\delta$ for all $k \in\{1, \ldots, N\}$, and let

$$
\lambda=V\left(P_{\delta}\right)^{-\frac{1}{n}}=\left(1-\left(c_{n} \delta^{n}+\ldots+c_{2} \delta^{2}\right)\right)^{-\frac{1}{n}} .
$$

From this and Equation (3.3), we have

$$
\begin{aligned}
\prod_{k=1}^{N}\left(h\left(\lambda P_{\delta}, u_{k}\right)-\xi\left(\lambda P_{\delta}\right) \cdot u_{k}\right)^{\gamma_{k}} & =\lambda \prod_{k=1}^{N}\left(h\left(P_{\delta}, u_{k}\right)-\xi\left(P_{\delta}\right) \cdot u_{k}\right)^{\gamma_{k}} \\
& =\lambda\left[\prod_{k=1}^{N}\left(h\left(P, u_{k}\right)-\xi\left(P_{\delta}\right) \cdot u_{k}\right)^{\gamma_{k}}\right]\left[\frac{h\left(P, u_{i_{0}}\right)-\xi\left(P_{\delta}\right) \cdot u_{i_{0}}-\delta}{h\left(P, u_{i_{0}}\right)-\xi\left(P_{\delta}\right) \cdot u_{i_{0}}}\right]^{\gamma_{i_{0}}} \\
& =\left[\prod_{k=1}^{N}\left(h\left(P, u_{k}\right)-\xi\left(P_{\delta}\right) \cdot u_{k}\right)^{\gamma_{k}}\right] \frac{\left(1-\frac{\delta}{h\left(P, u_{i_{0}}\right)-\xi\left(P_{\delta}\right) \cdot u_{i_{0}}}\right)^{\gamma_{i_{0}}}}{\left(1-\left(c_{n} \delta^{n}+\ldots+c_{2} \delta^{2}\right)\right)^{\frac{1}{n}}} \\
& \leq\left[\prod_{k=1}^{N}\left(h\left(P, u_{k}\right)-\xi\left(P_{\delta}\right) \cdot u_{k}\right)^{\gamma_{k}}\right] \frac{\left(1-\frac{\delta}{d_{0}}\right)^{\gamma_{i_{0}}}}{\left(1-\left(c_{n} \delta^{n}+\ldots+c_{2} \delta^{2}\right)\right)^{\frac{1}{n}}}
\end{aligned}
$$

where $d_{0}=d(P)$ is the diameter of $P$. Thus,

$$
\Phi_{\lambda P_{\delta}}\left(\xi\left(\lambda P_{\delta}\right)\right) \leq \Phi_{P}\left(\xi\left(P_{\delta}\right)\right)+B(\delta),
$$

where

$$
B(\delta)=\gamma_{i_{0}} \log \left(1-\frac{\delta}{d_{0}}\right)-\frac{1}{n} \log \left(1-\left(c_{n} \delta^{n}+\ldots+c_{2} \delta^{2}\right)\right)
$$


Obviously,

$$
B^{\prime}(\delta)=\gamma_{i_{0}} \frac{-1 / d_{0}}{1-\delta / d_{0}}+\frac{1}{n} \frac{n c_{n} \delta^{n-1}+\ldots+2 c_{2} \delta}{1-\left(c_{n} \delta^{n}+\ldots+c_{2} \delta^{2}\right)}<0,
$$

when the positive $\delta$ is small enough. From this and the fact that $B_{1}(0)=0$,

$$
B(\delta)<0
$$

when the positive $\delta$ is small enough.

From this and Equations (4.6), (4.7), (4.8), there exists a $\delta_{0}>0$ such that $P_{\delta_{0}} \in \mathcal{P}\left(u_{1}, \ldots, u_{N}\right)$ and

$$
\Phi_{\lambda_{0} P_{\delta_{0}}}\left(\xi\left(\lambda_{0} P_{\delta_{0}}\right)\right)<\Phi_{P}\left(\xi\left(P_{\delta_{0}}\right)\right) \leq \Phi_{P}(\xi(P))=\Phi_{P}(0)
$$

where $\lambda_{0}=V\left(P_{\delta_{0}}\right)^{-\frac{1}{n}}$. Let $P_{0}=\lambda_{0} P_{\delta_{0}}-\xi\left(\lambda_{0} P_{\delta_{0}}\right)$, then $P_{0} \in \mathcal{P}\left(u_{1}, \ldots, u_{N}\right), V\left(P_{0}\right)=1, \xi\left(P_{0}\right)=0$ and

$$
\Phi_{P_{0}}(0)<\Phi_{P}(0)
$$

This contradicts Equation (4.5). Therefore, $P \in \mathcal{P}_{N}\left(u_{1}, \ldots, u_{N}\right)$.

\section{Existence of the solution to the discrete logarithmic Minkowski Problem}

If $\mu$ is a Borel measure on $S^{n-1}$ and $\xi$ is a proper subspace of $\mathbb{R}^{n}$, it will be convenient to write $\mu_{\xi}$ for the restriction of $\mu$ to $S^{n-1} \cap \xi$. In this section, we prove the main result Theorem 1.5 of this paper based on the folowing idea. Let $\mu$ be discrete measure on $S^{n-1}, n \geq 2$, that is not concentrated on any closed hemisphere and satisfies the essential subspace concentration condition. If $\mu$ satisfies the strict essential subspace concentration inequality, then Lemma 4.7 yields that $\mu$ is a cone volume measure. Otherwise there exist complementary proper subspaces $\xi$ and $\xi^{\prime}$ such that $\operatorname{supp} \mu=S^{n-1} \cap\left(\xi \cup \xi^{\prime}\right)$, and $\mu_{\xi}$ and $\mu_{\xi}^{\prime}$ are not concentrated on any closed hemisphere of $\xi \cap S^{n-1}$ and $\xi^{\prime} \cap S^{n-1}$, respectively, and satisfy the essential subspace concentration condition. Therefore $\mu_{\xi}$ and $\mu_{\xi}^{\prime}$ are cone volume measures on $\xi \cap S^{n-1}$ and $\xi^{\prime} \cap S^{n-1}$, respectively, by induction on the dimension of the ambient space, which in turn imply that $\mu$ is a cone volume measure.

However, it is possible that $\operatorname{dim} \xi=1$. Therefore in order to execute the plan, we extend the notions occuring in Theorem 1.5 to $\mathbb{R}^{1}$. The role of a compact convex set containing the origin in its interior is played by some interval $K=[a, b]$ with $a<0$ and $b>0$, and closed hemispheres of $S^{0}=\{-1,1\}$ are $\{1\}$ and $\{-1\}$. The cone volume measure on $S^{0}$ associated to $K$ satisfies $V_{K}(\{-1\})=|a|$ and $V_{K}(\{1\})=b$. In addition, we say that a non-trivial measure $\mu$ on $S^{0}$ satisfies the essential subspace concentration inequality if it is not concentrated on any closed hemisphere; namely, if $\mu(\{-1\})>0$ and $\mu(\{1\})>0$. These notions are in accordance with Definition 1.3 because if $n=1$, then there is no subspace $\xi$ such that $0<\operatorname{dim} \xi<n$.

We note that the notion of strict essential subspace concentration inequality is defined and used only if the dimension $n \geq 2$.

The following lemma will be needed. The proof is the same that of Lemma 7.1 in [6].

Lemma 5.1. Suppose $n \geq 2, \mu$ is a discrete measure on $S^{n-1}$ that satisfies the essential subspace concentration condition. If $\xi$ is an essential linear subspace with respect to $\mu$ for which

$$
\mu\left(\xi \cap S^{n-1}\right)=\frac{1}{n} \mu\left(S^{n-1}\right) \operatorname{dim} \xi
$$

then $\mu_{\xi}$ satisfies the essential subspace concentration condition.

For even measures, the following lemma was stated for even measures as Lemma 7.2 in [6]. However, the proof in [6] does not use the property that the measure is even. 
Lemma 5.2. Let $\xi$ and $\xi^{\prime}$ be complementary subspaces in $\mathbb{R}^{n}$ with $0<\operatorname{dim} \xi<n$. Suppose $\mu$ is a Borel measure on $S^{n-1}$ that is concentrated on $S^{n-1} \cap\left(\xi \cup \xi^{\prime}\right)$, and so that

$$
\mu\left(\xi \cap S^{n-1}\right)=\frac{1}{n} \mu\left(S^{n-1}\right) \operatorname{dim} \xi .
$$

If $\mu_{\xi}$ and $\mu_{\xi^{\prime}}$ are cone-volume measures of convex bodies in the subspaces $\xi$ and $\xi^{\prime}$, then $\mu$ is the cone-volume measure of a convex body in $\mathbb{R}^{n}$.

In addition, we also need the following lemma.

Lemma 5.3. Suppose $\mu$ is a Borel measure on $S^{n-1}, n \geq 2$, that is not concentrated on any closed hemisphere, and $\mu$ concentrated on two complementary subspaces $\xi$ and $\xi^{\prime}$ of $\mathbb{R}^{n}$. Then, $\mu_{\xi}$ is not concentrated on any closed hemisphere of $\xi \cap S^{n-1}$ and $\mu_{\xi^{\prime}}$ is not concentrated on any closed hemisphere of $\xi^{\prime} \cap S^{n-1}$.

Proof. We only need prove that $\mu_{\xi}$ is not concentrated on any closed hemisphere of $\xi \cap S^{n-1}$.

Suppose $\mu_{\xi}$ is concentrated on a closed hemisphere, $C$, of $\xi \cap S^{n-1}$. Then, $\mu$ is concentrated on

$$
S^{n-1} \cap \operatorname{pos}\left\{C \cup \xi^{\prime}\right\} \text {. }
$$

However, $S^{n-1} \cap \operatorname{pos}\left\{C \cup \xi^{\prime}\right\}$ is a closed hemisphere of $S^{n-1}$. This contradicts the conditions of the lemma. Therefore, $\mu_{\xi}$ is not concentrated on any closed hemisphere of $\xi \cap S^{n-1}$.

Now, we have prepared enough to prove the main theorem of this paper.

Theorem 5.4. If $\mu$ is a discrete measure on $S^{n-1}, n \geq 1$ that is not concentrated on any closed hemisphere and satisfies the essential subspace concentration condition, then $\mu$ is the cone-volume measure of a polytope in $\mathbb{R}^{n}$.

Proof. We prove Theorem 5.4 by induction on the dimension $n \geq 1$. If $n=1$, then the theorem trivially holds, therefore let $n \geq 2$.

If $\mu$ satisfies the strict essential subspace concentration inequality, then $\mu$ is the cone-volume measure of a polytope in $\mathbb{R}^{n}$ according to Lemma 3.4 and Lemma 4.7.

Therefore we assume that there exists an essential subspace (with respect to $\mu$ ), $\xi$, of $\mathbb{R}^{n}$, and a subspace, $\xi^{\prime}$, of $\mathbb{R}^{n}$ such that $\xi, \xi^{\prime}$ are complementary subspaces of $\mathbb{R}^{n}$, $\mu$ concentrated on $S^{n-1} \cap\left\{\xi \cup \xi^{\prime}\right\}$ with

$$
\mu\left(S^{n-1} \cap \xi\right)=\frac{\operatorname{dim} \xi}{n} \mu\left(S^{n-1}\right) \text { and } \mu\left(S^{n-1} \cap \xi^{\prime}\right)=\frac{\operatorname{dim} \xi^{\prime}}{n} \mu\left(S^{n-1}\right) .
$$

From the fact that $\mu$ is not concentrated on a closed hemisphere and Lemma 5.3, we have, $\mu_{\xi}$ is not concentrated on a closed hemisphere of $S^{n-1} \cap \xi$, and $\mu_{\xi^{\prime}}$ is not concentrated on a closed hemisphere of $S^{n-1} \cap \xi^{\prime}$. By Lemma 5.1, $\mu_{\xi}$ satisfies the essential subspace concentration condition on $\xi \cap S^{n-1}$, and $\mu_{\xi^{\prime}}$ satisfies the essential subspace concentration condition on $\xi^{\prime} \cap S^{n-1}$. From the induction hypothesis, $\mu_{\xi}$ is the cone-volume measure of a convex body in $\xi \cap \mathbb{R}^{n}$, and $\mu_{\xi^{\prime}}$ is the cone-volume measure of a convex body in $\xi^{\prime} \cap \mathbb{R}^{n}$. By Lemma 5.2 , $\mu$ is the cone-volume measure of a convex body in $\mathbb{R}^{n}$. Since $\mu$ is discrete, $\mu$ is the cone-volume measure of a polytope in $\mathbb{R}^{n}$.

\section{NeW inequalities FOR CONE-VOLUme MEASURES}

In this section, we establish some inequalities for cone-volume measures.

The following example shows that the cone-volume measure of a convex body does not need to satisfy the essential subspace concentration condition with respect to essential linear subspace. 
Example 6.1. Let $u_{1}, \ldots, u_{n}$ be an orthonormal basis of $\mathbb{R}^{n}$, and let $W=\left\{x \in u_{1}^{\perp}:\left|x \cdot u_{i}\right| \leq 1, i=\right.$ $2, \ldots, n\}$ be an $(n-1)$-dimensional cube. For $r>0$ and $i=1, \ldots, n-1, \xi_{i}=\operatorname{lin}\left\{u_{1}, \ldots, u_{i}\right\}$ is an essential subspace for the cone-volume measure of the truncated pyramid $P_{r}=\left[-r u_{1}+r W, u_{1}+W\right]$. If $r>0$ is small, then $P_{r}$ approximates $\left[o, u_{1}+W\right]$, and thus

$$
V_{P_{r}}\left(\xi_{i} \cap S^{n-1}\right)>V_{P_{r}}\left(\left\{u_{1}\right\}\right)=V\left(\left[o, u_{1}+W\right]\right)>\frac{i}{n} V\left(P_{r}\right) .
$$

We next establish new inequalities for the cone-volume measures.

Lemma 6.2. If $K$ is a convex body in $\mathbb{R}^{n}, n \geq 3$, with $o \in \operatorname{Int}(K)$, then for $u \in S^{n-1}$

$$
V_{K}(\{u\})+V_{K}(\{-u\})+2(n-1) \sqrt{V_{K}(\{u\}) V_{K}(\{-u\})} \leq V(K),
$$

with equality if and only if $F(K,-u)$ is a translate of $F(K, u), K=[F(K, u), F(K,-u)]$, and $h(K, u)=h(K,-u)$.

In $\mathbb{R}^{2}$, we have

Lemma 6.3. If $K$ is a convex body containing the origin in its interior in $\mathbb{R}^{2}$, and $u \in S^{1}$, then

$$
\sqrt{V_{K}(\{u\})}+\sqrt{V_{K}(\{-u\})} \leq \sqrt{V(K)}
$$

with equality if and only if $K$ is a trapezoid with two sides parallel to $u^{\perp}$, and $u^{\perp}$ contains the intersection of the diagonals.

We obtain the following estimate from Lemma 6.2 and Lemma 6.3.

Corollary 6.4. If $K$ is a convex body in $\mathbb{R}^{n}, n \geq 2$ with o $\in \operatorname{Int}(K)$ and $u \in S^{n-1}$, then

$$
V_{K}(\{u\}) \cdot V_{K}(\{-u\}) \leq \frac{1}{4 n^{2}}(V(K))^{2},
$$

with equality if and only if $F(K,-u)$ is a translate of $F(K, u), K=[F(K, u), F(K,-u)]$, and $h(K, u)=h(K,-u)$.

We next prove Lemma 6.2 and Lemma 6.3 together.

Proof. For the case $|F(K, u)| \cdot|F(K,-u)|=0$, Lemma 6.2 and Lemma 6.3 are trivially true. Thus we prove Lemma 6.2 and Lemma 6.3 under the condition that $|F(K, u)| \cdot|F(K,-u)|>0$.

Let $V_{K}(\{u\})=\alpha>0$ and $V_{K}(\{-u\})=\beta>0$, let $h_{K}(u)=a$ and $h_{K}(-u)=b$, and for $0 \leq x \leq a+b$ let

$$
K_{x}=\left((a-x) u+u^{\perp}\right) \cap K .
$$

Since $K$ is a convex body,

$$
\frac{x}{a+b} F(K,-u)+\frac{a+b-x}{a+b} F(K, u) \subset K_{x} .
$$

From this and the Brunn-Minkowski inequality,

$$
\begin{aligned}
\left|K_{x}\right| & \geq\left|\frac{x}{a+b} F(K,-u)+\frac{a+b-x}{a+b} F(K, u)\right| \\
& =\left|\left(\frac{x}{a+b} F(K,-u)+\frac{a+b-x}{a+b} F(K, u)\right)_{u^{\perp}}\right| \\
& =\left|\frac{x}{a+b} F(K,-u)\right|_{u^{\perp}}+\left.\frac{a+b-x}{a+b} F(K, u)\right|_{u^{\perp}} \mid \\
& \geq\left(\left.\frac{x}{a+b}|F(K,-u)|_{u^{\perp}}\right|^{\frac{1}{n-1}}+\left.\frac{a+b-x}{a+b}|F(K, u)|_{u^{\perp}}\right|^{\frac{1}{n-1}}\right)^{n-1} \\
& =\left(\frac{x}{a+b}|F(K,-u)|^{\frac{1}{n-1}}+\frac{a+b-x}{a+b}|F(K, u)|^{\frac{1}{n-1}}\right)^{n-1},
\end{aligned}
$$


with equality if and only if $K_{x}=\frac{x}{a+b} F\left(u(K,-u)+\frac{a+b-x}{a+b} F(K, u)\right.$, and $\left.F(K,-u)\right|_{u^{\perp}}$ and $\left.F(K, u)\right|_{u^{\perp}}$ are homothetic.

Let $t=\frac{a+b-x}{a+b}$. From (6.3) and Fubini's formula,

$$
\begin{aligned}
V(K) & =\int_{0}^{a+b}\left|K_{x}\right| d x \\
& \geq \int_{0}^{a+b}\left(\frac{x}{a+b}|F(K,-u)|^{\frac{1}{n-1}}+\frac{a+b-x}{a+b}|F(K, u)|^{\frac{1}{n-1}}\right)^{n-1} d x \\
& =(a+b) \int_{0}^{1}\left(t|F(K, u)|^{\frac{1}{n-1}}+(1-t)|F(K,-u)|^{\frac{1}{n-1}}\right)^{n-1} d t \\
& =(a+b) \sum_{i=0}^{n-1}|F(K, u)|^{\frac{i}{n-1}}|F(K,-u)|^{\frac{n-1-i}{n-1}}\left(\begin{array}{c}
n-1 \\
i
\end{array}\right) \int_{0}^{1} t^{i}(1-t)^{n-1-i} d t \\
& =\frac{a+b}{n} \sum_{i=0}^{n-1}|F(K, u)|^{\frac{i}{n-1}}|F(K,-u)|^{\frac{n-1-i}{n-1}} .
\end{aligned}
$$

Let $S_{1}=|F(K, u)|$ and $S_{2}=|F(K,-u)|$. From (6.4) and the arithmetic-geometric inequality, we have

$$
\begin{aligned}
V(K) & =\frac{a+b}{n} \sum_{i=0}^{n-1} S_{1}^{\frac{i}{n-1}} S_{2}^{\frac{n-1-i}{n-1}} \\
& =\frac{a}{n} S_{1}+\frac{b}{n} S_{2}+\frac{1}{n} \sum_{i=1}^{n-1}\left(a S_{1}^{\frac{n-1-i}{n-1}} S_{2}^{\frac{i}{n-1}}+b S_{2}^{\frac{n-1-i}{n-1}} S_{1}^{\frac{i}{n-1}}\right) \\
& \geq \alpha+\beta+2(n-1) \sqrt{\alpha \beta} .
\end{aligned}
$$

Thus, we get (6.1) and (6.2).

From the equality conditions for $(6.3),(6.4)$ and the arithmetic-geometric inequality, we have, equality holds in (6.5) if and only if $\left.F(K, u)\right|_{u^{\perp}}$ and $\left.F(K,-u)\right|_{u^{\perp}}$ are homothetic, $K=[F(K, u), F(K,-u)]$, and

$$
\frac{a}{b}=\left(\frac{S_{1}}{S_{2}}\right)^{\frac{2 i-n+1}{n-1}}
$$

for all $1 \leq i \leq n-1$.

Therefore, equality holds in (6.2) $(n=2)$ if and only if $K$ is a trapezoid with two sides parallel to $u^{\perp}$, and $u^{\perp}$ contains the intersection of the diagonals.

When $n \geq 3$, (6.6) hold for $i=1, \ldots, n-1$. Thus, $\frac{a}{b}=\frac{S_{1}}{S_{2}}=1$. Therefore, equality holds in (6.1) if and only if $F(K,-u)$ is a translation of $F(K, u), K=[F(K, u), F(K,-u)]$, and $h_{K}(u)=$ $h_{K}(-u)$.

Funding This work was supported by the Hungarian Scientific Research Fund [109789 to K.J.B., 84233 to P.H.].

Acknowledgement We thank the unknown referees for many improvements.

\section{REFERENCES}

[1] A.D. Alexandrov, Existence and uniqueness of a convex surface with a given integral curvature. C. R. (Doklady) Acad. Sci. USSR (N.S.) 35, 131-134 (1942).

[2] B. Andrews, Gauss curvature flow: the fate of the rolling stones. Invent. Math. 138, 151-161 (1999). 
[3] B. Andrews, Classification of limiting shapes for isotropic curve flows. J. Amer. Math. Soc. 16, 443-459 (2003).

[4] F. Barthe, O. Guédon, S. Mendelson, A. Naor, A probabilistic approach to the geometry of the $l_{p}^{n}$-ball. Ann. of Probability 33, 480-513 (2005).

[5] K. Böröczky, M. Henk, Cone-volume measure and stability. submitted.

[6] J. Böröczky, E. Lutwak, D. Yang, G. Zhang, The logarithmic Minkowski problem. J. Amer. Math. Soc. 26, 831-852 (2013).

[7] J. Böröczky, E. Lutwak, D. Yang, G. Zhang, The log-Brunn-Minkowski inequality. Adv. Math. 231, 19741997 (2012).

[8] Böröczky, J., Lutwak, E., Yang, D., Zhang, G.: Affine images of isotropic measures, to appear in J. Diff. Geom.

[9] L. Caffarelli, Interior $W^{2, p}$-estimates for solutions of the Monge-Ampère equation. Ann. of Math. (2) 131, 135-150 (1990).

[10] S. Campi, P. Gronchi, The $L^{p}$-Busemann-Petty centroid inequality. Adv. Math. 167, 128-141 (2002).

[11] W. Chen, $L_{p}$ Minkowski problem with not necessarily positive data. Adv. Math. 201, 77-89 (2006).

[12] S.-Y. Cheng, S.-T. Yau, On the regularity of the solution of the $n$-dimensional Minkowski problem. Comm. Pure Appl. Math. 29, 495-561 (1976).

[13] K.-S. Chou, Deforming a hypersurface by its Gauss-Kronecker curvature. Comm. Pure Appl. Math. 38, 867-882 (1985).

[14] K.-S. Chou, X.-J. Wang, The $L_{p}$-Minkowski problem and the Minkowski problem in centroaffine geometry. Adv. Math. 205, 33-83 (2006).

[15] A. Cianchi, E. Lutwak, D. Yang, G. Zhang, Affine Moser-Trudinger and Morrey-Sobolev inequalities. Calc. Var. Partial Differential Equations 36, 419-436 (2009).

[16] M. Gage, R. Hamilton, The heat equation shrinking convex plane curves. J. Differential Geom. 23, 69-96 (1986).

[17] R.J. Gardner, Geometric Tomography, 2nd edition, Encyclopedia of Mathematics and its Applications. Cambridge University Press, Cambridge, 2006.

[18] P.M. Gruber, Convex and discrete geometry, Grundlehren der Mathematischen Wissenschaften, 336. Springer, Berlin, 2007.

[19] B. Grünbaum, convex polytope (graduate Texts in Mathematics), 2nd edition. Springer, 2003.

[20] B. Guan, P. Guan, Convex hypersurfaces of prescribed curvatures. Ann. of Math. (2) 156, 655-673 (2002).

[21] P. Guan, C.-S. Lin, On equation $\operatorname{det}\left(u_{i j}+\delta_{i j} u\right)=u^{p} f$ on $S^{n}$. (preprint).

[22] P. Guan, X. Ma, The Christoffel-Minkowski problem I: Convexity of solutions of a Hessian equation. Invent. Math. 151, 553-577 (2003).

[23] C. Haberl, Minkowski valuations intertwining with the special linear group. J. Eur. Math. Soc. 14, 1565-1597 (2012).

[24] C. Haberl, E. Lutwak, D. Yang, G. Zhang, The even Orlicz Minkowski problem. Adv. Math. 224, 2485-2510 (2010).

[25] C. Haberl, L. Parapatits, Valuations and surface area measures. J. Reine Angew. Math. 687, 225-245 (2014).

[26] C. Haberl, L. Parapatits, The centro-affine Hadwiger theorem. J. Amer. Math. Soc. 27, 685-705 (2014).

[27] C. Haberl, F. Schuster, General $L_{p}$ affine isoperimetric inequalities. J. Differential Geom. 83, 1-26 (2009).

[28] C. Haberl, F. Schuster, Asymmetric affine $L_{p}$ Sobolev inequalities. J. Funct. Anal. 257, 641-658 (2009).

[29] C. Haberl, F. Schuster, J. Xiao, An asymmetric affine Pólya-Szegö principle. Math. Ann. 352, 517-542 (2012).

[30] B. He, G. Leng \& K, Li, Projection problems for symmetric polytopes. Adv. Math. 207, 73-90 (2006).

[31] M. Henk, E. Linke, Cone-volume measures of polytopes. Adv. Math. 253, 50-62 (2014).

[32] M. Henk, A. Schürmann \& J.M. Wills, Ehrhart polynomials and successive minima. Mathematika 52(1-2):1162005.

[33] C. Hu, X. Ma, C. Shen, On the Christoffel-Minkowski problem of Firey's p-sum. Calc. Var. Partial Differential Equations. 21, 137-155 (2004).

[34] Y. Huang, Q. Lu, On the regularity of the $L_{p}$-Minkowski problem. Adv. in Appl. Math. 50, 268-280 (2013).

[35] D. Hug, E. Lutwak, D. Yang, G. Zhang, On the $L_{p}$ Minkowski problem for polytopes. Discrete Comput. Geom. 33, 699-715 (2005).

[36] G. Huisken, Flow by mean curvature of convex surfaces into spheres. J. Differential Geom. 20, 237-266 (1984). 
[37] M.N. Ivaki, A flow approach to the $L_{-2}$ Minkowski problem. Advances in Applied Mathematics, 50 (2013), 445-464.

[38] M.Y. Jiang, Remarks on the 2-dimensional $L_{p}$-Minkowski problem. Adv. Nonlinear Stud. 10, 297-313 (2010).

[39] D. Klain, The Minkowski problem for polytopes. Adv. Math. 185, 270-288 (2004).

[40] H. Lewy, On differential geometry in the large. I. Minkowski problem. Trans. Amer. Math. Soc. 43, 258-270 (1938).

[41] J. Lu, X.-J. Wang, Rotationally symmetric solution to the $L_{p}$-Minkowski problem. J. Differential Equations. 254, 983-1005 (2013).

[42] M. Ludwig, Ellipsoids and matrix-valued valuations. Duke Math. J. 119, 159-188 (2003).

[43] M. Ludwig, General affine surface areas. Adv. Math. 224, 2346-2360 (2010).

[44] M. Ludwig, M. Reitzner, A classification of SL(n) invariant valuations. Ann. of Math. (2) 172, 1219-1267 (2010).

[45] E. Lutwak, The Brunn-Minkowski-Firey theory. I. Mixed volumes and the Minkowski problem. J. Differential Geom. 38, 131-150 (1993).

[46] E. Lutwak, V. Oliker, On the regularity of solutions to a generalization of the Minkowski problem. J. Differential Geom. 41, 227-246 (1995).

[47] E. Lutwak, D. Yang, G. Zhang, $L_{p}$ affine isoperimetric inequalities. J. Differential Geom. 56, 111-132 (2000).

[48] E. Lutwak, D. Yang, G. Zhang, A new ellipsoid associated with convex bodies. Duke Math. J. 104, 375-390 (2000).

[49] E. Lutwak, D. Yang, G. Zhang, The Cramer-Rao inequality for star bodies. Duke Math. J. 112, 59-81 (2002).

[50] E. Lutwak, D. Yang, G. Zhang, Sharp affine $L_{p}$ Sobolev inequalities. J. Differential Geom. 62, 17-38 (2002).

[51] E. Lutwak, D. Yang, G. Zhang, On the $L_{p}$-Minkowski problem. Trans. Amer. Math. Soc. 356, 4359-4370 (2004).

[52] E. Lutwak, D. Yang, G. Zhang, Volume inequalities for subspaces of $L_{p}$. J. Differential Geom. 68, 159-184 (2004).

[53] E. Lutwak, G. Zhang, Blaschke-Santaló inequalities. J. Differential Geom. 47, 1-16 (1997).

[54] H. Minkowski, Allgemeine Lehrsätze über die konvexen Polyeder. Gött. Nachr. 1897, 198-219 (1897).

[55] A. Naor, The surface measure and cone measure on the sphere of $l_{p}^{n}$. Trans. Amer. Math. Soc. 359, 1045-1079 (2007).

[56] A. Naor, D. Romik, Projecting the surface measure of the sphere of $l_{p}^{n}$. Ann. Inst. H. Poincaré Probab. Statist. 39, 241-261 (2003).

[57] L. Nirenberg, The Weyl and Minkowski problems in differential geometry in the large. Comm. Pure and Appl. Math. 6, 337-394 (1953).

[58] G. Paouris, Concentration of mass on convex bodies. Geom. Funct. Anal. 16, 1021-1049 (2006).

[59] G. Paouris, E. Werner, Relative entropy of cone measures and $L_{p}$ centroid bodies. Proc. London Math. Soc. 104, 253-286 (2012).

[60] A.V. Pogorelov, The Minkowski multidimensional problem. V.H. Winston \& Sons, Washington, D.C, 1978.

[61] R. Schneider, Convex bodies: the Brunn-Minkowski theory, Encyclopedia of Mathematics and its Applications (Second Edition). Cambridge University Press, Cambridge, 2014.

[62] A. Stancu, The discrete planar $L_{0}$-Minkowski problem. Adv. Math. 167, 160-174 (2002).

[63] A. Stancu, On the number of solutions to the discrete two-dimensional $L_{0}$-Minkowski problem. Adv. Math. 180, 290-323 (2003).

[64] A. Stancu, Centro-affine invariants for smooth convex bodies. Int. Math. Res. Not. 2012, 2289-2320 (2012).

[65] A. C. Thompson, Minkowski geometry, Encyclopedia of Mathematics and its Applications. Cambridge University Press, Cambridge, 1996.

[66] T. Wang, The affine Sobolev-Zhang inequality on $B V\left(\mathbb{R}^{n}\right)$. Adv. Math. 230, 2457-2473 (2012).

[67] G. Xiong, Extremum problems for the cone volume functional for convex polytopes. Adv. Math. 225, 32143228 (2010).

[68] G. Zhang, The affine Sobolev inequality. J. Differential Geom. 53, 183-202 (1999).

[69] G. Zhu, The logarithmic Minkowski problem for polytopes. Adv. Math. 262, 909-931 (2014).

[70] G. Zhu, The centro-affine Minkowski problem for polytopes. J. Differential Geom. (in press).

[71] G. Zhu, The $L_{p}$ Minkowski problem for polytopes for $0<p<1$. (submitted). 
Alfréd Rényi Institute of Mathematics, Hungarian Academy of Sciences, Reltanoda u. 1315, H-1053 Budapest, Hungary, and Department of Mathematics, Central European University, NAdor u 9, H-1051, Budapest, Hungary,BOroczKy.Karoly.J@REnyi.MTA.Hu

Department of Mathematics, Central European University, Nador u 9, H-1051, Budapest, HunGARY,HEGEDUSP@CEU.EDU

Department of Mathematics, Polytechnic School of Engineering, New York University, Six Metrotech, Brooklin, NY 11201, USA,GUANGXian.Zhu@Gmail.Com 